\title{
ASYMPTOTIC BEHAVIOR OF A BAM NEURAL NETWORK WITH DELAYS OF DISTRIBUTED TYPE
}

\author{
S. Othmani ${ }^{1, *}$, N.-E. TataR ${ }^{2}$ And A. Khemmoudj ${ }^{1}$
}

\begin{abstract}
In this paper, we examine a Bidirectional Associative Memory neural network model with distributed delays. Using a result due to Cid [J. Math. Anal. Appl. 281 (2003) 264-275], we were able to prove an exponential stability result in the case when the standard Lipschitz continuity condition is violated. Indeed, we deal with activation functions which may not be Lipschitz continuous. Therefore, the standard Halanay inequality is not applicable. We will use a nonlinear version of this inequality. At the end, the obtained differential inequality which should imply the exponential stability appears 'state dependent'. That is the usual constant depends in this case on the state itself. This adds some difficulties which we overcome by a suitable argument.
\end{abstract}

Mathematics Subject Classification. 34A40, 92B20, 34A34, 34C11, 34D05, 34D20.

Received April 11, 2020. Accepted April 24, 2021.

\section{INTRODUCTION}

Recently, artificial neural networks have received a lot of attention in sciences and engineering, more specifically, in economics, biology, medicine, gas and petroleum industry $[1,25,40]$ and in many other disciplines. They are derived in the context of Artificial Intelligence. Their standard design is based on three neural layers known as the input layer, output layer, and some hidden layers. In each layer, the artificial neurons are connected to all neurons in the surrounding layers via artificial synapses in a similar manner to biological neurons. The main operations of neural networks are summed up to help predicting, classifying, recognizing models and solving optimization problems. The Recurrent neural networks encompass Hopfield neural networks, Cohen-Grossberg neural networks, Bidirectional Associative Memory neural networks, Cellular networks and many others.

In 1983, Hopfield suggested a single-layer self-associative Hebbian correlator model as follows

$$
x_{i}^{\prime}(t)=-c_{i} x_{i}(t)+\sum_{j=1}^{n} a_{i j} f_{j}\left(x_{j}(t)\right), \quad t>0, \quad i=1,2, \ldots, n
$$

Keywords and phrases: Exponential stability, BAM neural network, distributed delay, nonlinear Halanay inequality.

${ }^{1}$ Laboratory of SDG, Faculty of Mathematics, University of Science and Technology Houari Boumedienne, P.O. Box 32, El-Alia 16111, Bab Ezzouar, Algiers, Algeria.

2 Department of Mathematics and Statistics, King Fahd University of Petroleum and Minerals, 31261 Dhahran, Saudi Arabia.

* Corresponding author: sothmani@usthb.dz 
After a few years, Kosko introduced an expansion of Hopfield neural networks named bidirectional associative memory (BAM) neural network [13-15]. It is used in pattern recognition, signal and image processing, optimization problems, and automatic control [21]. Its architecture relies on two interconnected hidden layers of neurons that have no links in a single layer. It is described by the system

$$
\begin{cases}x_{i}^{\prime}(t)=-c_{i} x_{i}(t)+\sum_{j=1}^{m} a_{j i} f_{j}\left(y_{j}(t)\right)+I_{i}, & i=1,2, \ldots, n, \\ y_{j}^{\prime}(t)=-\bar{c}_{j} y_{j}(t)+\sum_{i=1}^{n} \bar{a}_{i j} g_{i}\left(x_{i}(t)\right)+J_{j}, & j=1,2, \ldots, m .\end{cases}
$$

In applications, it is frequent that, oscillations, divergences, chaos, and bifurcations affect negatively structures. One of the causes may be the occurrence of delays. Owing to the existence of a multitude of parallel paths with axons of different sizes and lengths, neural networks typically have a spatial extent, and therefore a distribution of propagation delays over a time span occurs [19, 26, 41].

Due to the increasing interest in the asymptotic behavior of solutions for designing neural networks, researchers have recently addressed the stability of delayed neural networks (see for instance $[5,8,10,12,16$ $18,20,23,24,38,39]$ and references therein). In [18, 38, 39], a set of sufficient conditions based on the system parameters guaranteeing the exponential stability of various retarded BAM neural network models was derived by analytical techniques and Lyapunov functionals. In addition, the authors in [5, 10, 17, 20, 23] obtained some LMI-dependent sufficient conditions ensuring either the exponential or asymptotic stability of BAM neural networks involving delays, via Karasovski Lyapunov functionals and analytical inequalities. In [8, 16, 24], the asymptotic stability of a class of delayed BAM neural networks was investigated utilizing the LMI approach, Lyapunov functionals, and analytical inequalities. Through a combination of the graph-theoretic approach, degree theory, and Lyapunov functionals, the exponential stability of a BAM neural network with delays was established in [12].

On the other hand, one of the basic components of artificial neural networks involves activation functions, linking the inputs to the outputs of the networks. Generally, the activation function of hidden neurons introduces a degree of nonlinearity that is of significant value in most applications of artificial neural networks. At first, such functions were assumed bounded, smooth, and monotonic [6, 36, 37]. For example, we can cite the threshold function, the piecewise linear function, and the sigmoid function. Thereafter, these conditions were eased somewhat to be of Lipschitz type, which is commonly considered in the existing literature [12, 18, 20, 23, 24, 39]. In view of the importance of non-Lipschitz activation functions in implementations [15], a relaxation of the Lipschitz condition is necessary. This has motivated some researchers to consider discontinuous functions and Hölder-type functions, one can refer to $[2,3,7,11,27-33,35]$.

As is well known, Cauchy problems described by

$$
\left\{\begin{array}{l}
x^{\prime}(t)=f(t, x(t)) \\
x\left(t_{0}\right)=x_{0}
\end{array}\right.
$$

have local solutions under the continuity of the function $f$ in a neighborhood of $\left(t_{0}, x_{0}\right)$. This can shown by the theorem of Peano, whilst the uniqueness of the solution is guaranteed under the Lipschitz continuity with respect to the second argument. Weaker conditions have been considered in several papers by Nagumo, Perron, Osgood, Kamke, Tonelli, and many others (see for instance [9]). In particular, Nagumo and Osgood proved the uniqueness of the solution under the condition

$$
|f(t, x)-f(t, y)| \leq \phi(|x-y|), \quad t>0,
$$


where $\phi(u)$ is a nondecreasing function of 'continuity-modulus' type satisfying the Osgood criterion: $\phi:[0, \infty) \rightarrow$ $[0, \infty)$, with $\phi(0)=0$ and $\phi(u)>0$ for $u>0$ and

$$
\int_{0}^{\delta} \frac{\mathrm{d} u}{\phi(u)}=\infty, \quad \delta>0 .
$$

In case one of the components of the function $f$ fails to be Lipschitz continuous and $f$ is Lipschitz continuous with respect to all the other remaining components including the first one $t$, Cid [4] succeeded in proving the uniqueness of the solution for a system of differential equations.

Motivated by the discussions above, in this paper, we examine the exponential stability of a BAM neural network model with distributed delays. Our first result is based on a nonlinear Halanay inequality, whilst the second one is proved in a direct fashion. Compared to previous literature, we deal here with less restrictive assumptions on the activation functions, which allows a larger class than the ones considered so far. More precisely, we treat non-linear and non-Lipschitz continuous activation functions, satisfying

$$
|\phi(t, x)-\phi(t, y)| \leq \psi(|x-y|)=|x-y| \tilde{\phi}(|x-y|), t>0
$$

for some non-decreasing function $\tilde{\phi}$.

This paper is arranged as follows: In Section 2, we introduce some notation, definitions, and technical lemmas, while Section 3 contains our exponential stability results proven with the help of a non-linear Halanay inequality. Numerical illustrations to confirm the obtained results are given in Section 4.

\section{MODEL DESCRIPTION AND PRELIMINARIES}

In this paper, we consider the following BAM neural network with continuously distributed delays, for $i=1,2, \ldots, n, j=1,2, \ldots, m$

$$
\left\{\begin{array}{l}
x_{i}^{\prime}(t)=-c_{i}\left(x_{i}(t)\right)+\sum_{j=1}^{m} a_{j i} f_{1 j}\left(y_{j}(t)\right)+\sum_{j=1}^{m} d_{j i} \int_{0}^{\infty} k_{j i}(s) f_{2 j}\left(y_{j}(t-s)\right) \mathrm{d} s+I_{i}, \quad t>0 \\
y_{j}^{\prime}(t)=-r_{j}\left(y_{j}(t)\right)+\sum_{i=1}^{n} \bar{a}_{i j} g_{1 i}\left(x_{i}(t)\right)+\sum_{i=1}^{n} \bar{d}_{i j} \int_{0}^{\infty} h_{i j}(s) g_{2 i}\left(x_{i}(t-s)\right) \mathrm{d} s+J_{j}, \quad t>0 \\
x_{i}(t)=\phi_{i}(t), \quad t \leq 0, \\
y_{j}(t)=\varphi_{j}(t), \quad t \leq 0,
\end{array}\right.
$$

where $n$ and $m$ denote the number of neurons in the first layer $L_{x}$ and in the second layer $L_{y}$, respectively; $x_{i}$ is the state of the $i$ th neuron in $L_{x}$ and $y_{j}$ is the state of the $j$ th neuron in $L_{y} ; c_{i}$ and $r_{j}$ refer to the charging time functions of the $i$ th and the $j$ th neuron, respectively; $a_{j i}, \bar{a}_{i j}, d_{j i}$ and $\bar{d}_{i j}$ correspond to the constants accounting for the synaptic connection strengths between neurons; $f_{l j}$ and $g_{l i}$ for $l=1,2$ represent the activation functions of the $i$ th state and the $j$ th state, respectively; $k_{j i}$ and $h_{i j}$ account for the delay kernel functions; $\phi_{i}$ and $\varphi_{j}$ are the history functions of the $i$ th state and the $j$ th state, respectively; $I_{i}$ and $J_{j}$ stand for the external inputs of the $i$ th and the $j$ th neuron.

Throughout this paper, we assume that

(A1) The functions $c_{i}$ and $r_{j}$ are monotone increasing continuous functions and there exist constants $\beta_{i}>0$ and $\bar{\beta}_{j}>0$ such that

$$
\frac{c_{i}(x)-c_{i}(y)}{x-y} \geq \beta_{i}, i=1,2, \ldots, n, \text { for all } x, y \in \mathbb{R} \text { with } x \neq y,
$$




$$
\frac{r_{j}(x)-r_{j}(y)}{x-y} \geq \bar{\beta}_{j}, j=1,2, \ldots, m, \text { for all } x, y \in \mathbb{R} \text { with } x \neq y .
$$

We denote by $\beta=\min _{1 \leq i \leq n} \beta_{i}, \bar{\beta}=\min _{1 \leq j \leq m} \bar{\beta}_{j}$.

This means that the charging time functions $c_{i}$ and $r_{j}$ are "superlinear". With these assumptions, the first two terms in the right hand sides of (2.1) are indeed dissipative terms (see the first two terms in the right hand sides of (3.4) and (3.5) below).

(A2) The initial data $\phi_{i}$ and $\varphi_{j}, i=1,2, \ldots, n, j=1,2, \ldots, m, t \leq 0$ are real-valued continuous functions.

(A3) The delay kernels $k_{j i}$ and $h_{i j}$ are nonnegative continuous functions such that for $i=1,2, \ldots, n, j=$ $1,2, \ldots, m$,

$$
\int_{0}^{\infty} e^{\alpha_{0} s} k_{j i}(s) \mathrm{d} s<\infty, \quad \int_{0}^{\infty} e^{\alpha_{0} s} h_{i j}(s) \mathrm{d} s<\infty, \quad \alpha_{0}>0 .
$$

The significance of these conditions is that the kernels are "subexponential". Roughly, they assert that the densities in the cells are decreasing in time. This is in line with the fading memory principle. Simple examples are the exponentially decaying kernels.

Next, we present some definitions and necessary lemmas for our main results.

Definition 2.1. [4] The function $f: U \subset \mathbb{R}^{n+1} \rightarrow \mathbb{R}^{n}, U$ open set, is said to be Lipschitz continuous when fixing a component $i_{0} \in\{0,1, \ldots, n\}$ if there exists a constant $L>0$ such that

$$
\begin{aligned}
& \left\|f\left(y_{0}, \ldots, z_{i_{0}}, \ldots, y_{n}\right)-f\left(\bar{y}_{0}, \ldots, z_{i_{0}}, \ldots, \bar{y}_{n}\right)\right\|_{\infty} \\
& \leq L\left\|\left(y_{0}, \ldots, y_{i_{0}-1}, y_{i_{0}+1}, \ldots, y_{n}\right)-\left(\bar{y}_{0}, \ldots, \bar{y}_{i_{0}-1}, \bar{y}_{i_{0}+1}, \ldots, \bar{y}_{n}\right)\right\|_{\infty}
\end{aligned}
$$

for all $\left(y_{0}, \ldots, z_{i_{0}}, \ldots, y_{n}\right),\left(\bar{y}_{0}, \ldots, z_{i_{0}}, \ldots, \bar{y}_{n}\right) \in U$.

Definition 2.2. We say that the system (1) is globally exponentially stable, if for any two solutions $\left(x_{i}(t), y_{j}(t)\right)$ and $\left(\bar{x}_{i}(t), \bar{y}_{j}(t)\right)$ (with $\left(\phi_{i}(t), \varphi_{j}(t)\right)$ and $\left(\bar{\phi}_{i}(t), \bar{\varphi}_{j}(t)\right)$ as initial data), there exist two positive constants $M$ and $\nu$ such that

$$
\|x(t)-\bar{x}(t)\|+\|y(t)-\bar{y}(t)\| \leq M(\|\phi(t)-\bar{\phi}(t)\|+\|\varphi(t)-\bar{\varphi}(t)\|) e^{-\nu t}, \quad t \geq 0 .
$$

If there exists a unique equilibrium $\left(x_{i}^{*}, y_{j}^{*}\right), i=1, \ldots, n, j=1, \ldots, m$, that is a solution of the system

$$
\left\{\begin{array}{l}
c_{i}\left(x_{i}^{*}\right)=\sum_{j=1}^{m} a_{j i} f_{1 j}\left(y_{j}^{*}\right)+\sum_{j=1}^{m} d_{j i} \int_{0}^{\infty} k_{j i}(s) f_{2 j}\left(y_{j}^{*}\right) \mathrm{d} s+I_{i}, \\
r_{j}\left(y_{j}^{*}\right)=\sum_{i=1}^{n} \bar{a}_{i j} g_{1 i}\left(x_{i}^{*}\right)+\sum_{i=1}^{n} \bar{d}_{i j} \int_{0}^{\infty} h_{i j}(s) g_{2 i}\left(x_{i}^{*}\right) \mathrm{d} s+J_{j},
\end{array}\right.
$$

then we get the usual exponential stability of this equilibrium.

The above properties are local if they hold only nearby the initial data.

Definition 2.3. Let $f: \mathbb{R} \rightarrow \mathbb{R}$ be a continuous function. The upper right Dini derivative $D^{+} f(t)$ is defined as

$$
D^{+} f(t)=\varlimsup_{h \rightarrow 0^{+}} \frac{1}{h}(f(t+h)-f(t)) .
$$

Let $f:=\left(f_{1}, f_{2}, \ldots, f_{n}\right)$ and $f_{i_{0}}$ is one of the components of $f$, where $i_{0} \in\{1,2, \ldots, n\}$.

The main result of Cid [4] is given below. 
Theorem 2.4. For an open set $U \subset \mathbb{R}^{n+1}$, let $f: U \subset \mathbb{R}^{n+1} \rightarrow \mathbb{R}^{n}$, and $\left(t_{0}, x_{0}\right) \in U$. Assume that $f$ is continuous and locally Lipschitz continuous when fixing a component $i_{0} \in\{0,1, \ldots, n\}$. If either $i_{0}=0$ or $f_{i_{0}}\left(t_{0}, x_{0}\right) \neq 0$, then there exists $a>0$ such that (1.1) has a unique solution in $\left[t_{0}-a, t_{0}+a\right]$.

Lemma 2.5. [22] Let $u: \mathbb{R} \rightarrow[0, \infty)$ satisfy the inequality

$$
u^{\prime}(t) \leq-a u(t)+b \int_{0}^{\infty} k(s) u(t-s) \mathrm{d} s, t>t_{0},
$$

where $a, b>0$ and $k$ be a nonnegative piecewise continuous function satisfying $\int_{0}^{\infty} k(s) e^{\alpha_{0} s} \mathrm{~d} s<\infty$ for some $\alpha_{0}>0$. If $b \int_{0}^{\infty} k(s) \mathrm{d} s<a$, then there is a positive constant $\alpha$ such that $0<\alpha<\alpha_{0}$ and

$$
u(t) \leq \bar{u}\left(t_{0}\right) e^{-\alpha\left(t-t_{0}\right)}, \quad t>t_{0},
$$

where $\bar{u}\left(t_{0}\right)=\sup _{-\infty<\sigma \leq t_{0}} u(\sigma)$.

In order to prove the first exponential stability result, we appeal to a nonlinear Halanay inequality.

Lemma 2.6. Nonlinear Halanay Inequality Let $z(t)$ be a nonnegative differentiable function satisfying

$$
\left\{\begin{array}{l}
z^{\prime}(t) \leq-a z(t)+f(z(t))+\int_{-\infty}^{t} k(t-s) g(z(s)) \mathrm{d} s, t>0 \\
z(t)=\varphi(t),-\infty<t \leq 0
\end{array}\right.
$$

where

$$
f(z) \leq z \tilde{f}(z), g(z) \leq z \tilde{g}(z)
$$

for some nonnegative continuous functions $\tilde{f}$ and $\tilde{g}$ with $\tilde{f}(0)=\tilde{g}(0)=0$. The kernel $k$ is a nonnegative continuous function such that $\int_{0}^{t} k(s) e^{\alpha_{0} s} \mathrm{~d} s<\infty$ for some $\alpha_{0}>0$. Then, there exist $\nu>0$ and $\alpha$ such that $0<\alpha<\alpha_{0}$ and

$$
z(t) \leq \bar{z}(0) e^{-\alpha t}, t \geq 0
$$

when

$$
\bar{z}(0):=\sup _{-\infty<t \leq 0} \varphi(t) \leq \nu
$$

Proof. Let $\nu$ be a real number such that $\tilde{g}(\nu) \int_{0}^{\infty} k(s) \mathrm{d} s<a-\tilde{f}(\nu)$. In view of the condition (2.3), the functions $\tilde{f}(z)$ and $\tilde{g}(z)$ can be non-decreasing. As $\tilde{f}(0)=\tilde{g}(0)=0$, we see that $\nu>0$. Assume that $\bar{z}(0)=\sup _{-\infty<t \leq 0} \varphi(t)<$ $\nu$ and that $z(s) \leq \nu$ for all $0 \leq s \leq T$. Then, in light of this assumption and (2.2), we find

$$
\begin{aligned}
z^{\prime}(t) & \leq-a z(t)+z(t) \tilde{f}(z(t))+\int_{-\infty}^{t} k(t-s) z(s) \tilde{g}(z(s)) \mathrm{d} s \\
& \leq-a z(t)+z(t) \tilde{f}(\nu)+\tilde{g}(\nu) \int_{-\infty}^{t} k(t-s) z(s) \mathrm{d} s
\end{aligned}
$$




$$
\leq-(a-\tilde{f}(\nu)) z(t)+\tilde{g}(\nu) \int_{-\infty}^{t} k(t-s) z(s) \mathrm{d} s, t \leq T
$$

We can apply Lemma 2.5 to (2.6) in case $\int_{0}^{t} k(s) e^{\alpha_{0} s} \mathrm{~d} s<\infty$ for some $\alpha_{0}>0$, to arrive at

$$
z(t) \leq \bar{z}(0) e^{-\alpha t}
$$

on $[0, T]$. Assume that there exists a $T^{*}$ such that $0<T^{*} \leq T$ and $z\left(T^{*}\right)=\nu$. Then

$$
\nu \leq \bar{z}(0) e^{-\alpha T^{*}}<\nu
$$

which is a contradiction. Therefore, by repeating the process, we obtain

$$
z(t) \leq \bar{z}(0) e^{-\alpha t}, \quad t \geq 0 .
$$

The proof is complete.

This result has been proved in [34] (submitted). For the sake of completeness, we reproduced the proof here.

\section{ExPONENTIAL STABILITY}

In this section, we establish a local exponential stability result of system (2.1). As stated in Theorem 3.1, our result is valid for solutions having close enough initial data. To this end, we need the following assumption on the activation functions.

(A4) The functions $f_{l j}$ and $g_{l i}$ are Lipschitz continuous when fixing a component $i_{0} \in\{0,1, \ldots, n\}$ and $j_{0} \in\{0,1, \ldots, m\}$ with the Lipschitz constants $L_{l j}$ and $M_{l i}, l=1,2, i=1,2, \ldots, n, j=1,2, \ldots, m$.

In the sense of the definition above, the existence of a local solution is guaranteed by Theorem 2.4. We can extend our solution to be global by establishing a priori estimates.

(A5) Assume that the functions $f_{l j_{0}}$ and $g_{l i_{0}}, l=1,2$ satisfy

$$
\left|f_{l j_{0}}\left(y_{j_{0}}\right)-f_{l j_{0}}\left(\bar{y}_{j_{0}}\right)\right| \leq\left|y_{j_{0}}-\bar{y}_{j_{0}}\right| \tilde{f}_{l j_{0}}\left(\left|y_{j_{0}}-\bar{y}_{j_{0}}\right|\right), l=1,2, y_{j_{0}}, \bar{y}_{j_{0}} \in \mathbb{R}
$$

and

$$
\left|g_{l i_{0}}\left(x_{i_{0}}\right)-g_{l i_{0}}\left(\bar{x}_{i_{0}}\right)\right| \leq\left|x_{i_{0}}-\bar{x}_{i_{0}}\right| \tilde{g}_{l i_{0}}\left(\left|x_{i_{0}}-\bar{x}_{i_{0}}\right|\right), l=1,2, x_{i_{0}}, \bar{x}_{i_{0}} \in \mathbb{R}
$$

where $\tilde{f}_{l j_{0}}$ and $\tilde{g}_{l i_{0}}$ are nonnegative, non-decreasing and continuous functions such that $\tilde{f}_{l j_{0}}(0)=\tilde{g}_{l i_{0}}(0)=0$.

This assumption avoids and relaxes the classical Lipschitz condition imposed on the activation functions. As a simple example, the pulse-code signal function (average of sampled pulses with an exponential weight) is not Lipschitz continuous.

(A6) The dominance property

$$
\left(\max _{i \neq i_{0}} M_{2 i}+\max _{j \neq j_{0}} L_{2 j}\right) \int_{0}^{\infty} H(t) \mathrm{d} t<\min \left\{\beta-\sum_{i=1}^{n} \sum_{j \neq j_{0}} L_{1 j}\left|a_{j i}\right|, \bar{\beta}-\sum_{j=1}^{m} \sum_{i \neq i_{0}} M_{1 i}\left|\bar{a}_{i j}\right|\right\},
$$

where

$$
H(t)=\sum_{i=1}^{n} \sum_{j=1}^{m} H_{i j}(t), \quad H_{i j}(t)=\sup _{t \geq 0}\left\{\left|d_{j i}\right| k_{j i}(t),\left|\bar{d}_{i j}\right| h_{i j}(t)\right\}, 1 \leq i \leq n, 1 \leq j \leq m
$$

holds. 
This assumption is a natural one, it has been always assumed in such cases. It guarantees the dissipativity of the system as a whole. Indeed, it states that the passive decay rates dominate the synaptic connection strengths.

Notice that, denoting by $u_{i}(t)=x_{i}(t)-\bar{x}_{i}(t), v_{j}(t)=y_{j}(t)-\bar{y}_{j}(t), u(t)=\sum_{i=1}^{n}\left|u_{i}(t)\right|$ and $v(t)=\sum_{j=1}^{m}\left|v_{j}(t)\right|$, we infer

$$
\left\{\begin{array}{l}
u_{i}^{\prime}(t)=-\tilde{c}_{i}\left(u_{i}(t)\right)+\sum_{j=1}^{m} a_{j i} \tilde{f}_{1 j}\left(v_{j}(t)\right)+\sum_{j=1}^{m} d_{j i} \int_{0}^{\infty} k_{j i}(s) \tilde{f}_{2 j}\left(v_{j}(t-s)\right) \mathrm{d} s, t>0, i=1,2, \ldots, n, \\
v_{j}^{\prime}(t)=-\tilde{r}_{j}\left(v_{j}(t)\right)+\sum_{i=1}^{n} \bar{a}_{i j} \tilde{g}_{1 i}\left(u_{i}(t)\right)+\sum_{i=1}^{n} \bar{d}_{i j} \int_{0}^{\infty} h_{i j}(s) \tilde{g}_{2 i}\left(u_{i}(t-s)\right) \mathrm{d} s, t>0, j=1,2, \ldots, m, \\
u_{i}(t)=\tilde{\phi}_{i}(t):=\phi_{i}(t)-\bar{\phi}_{i}(t), t \leq 0, i=1,2, \ldots, n \\
v_{j}(t)=\tilde{\varphi}_{j}(t):=\varphi_{j}(t)-\bar{\varphi}_{j}(t), t \leq 0, j=1,2, \ldots, m
\end{array}\right.
$$

where

$$
\begin{gathered}
\tilde{c}_{i}\left(u_{i}(t)\right)=c_{i}\left(x_{i}(t)\right)-c_{i}\left(\bar{x}_{i}(t)\right), \quad \tilde{r}_{j}\left(v_{j}(t)\right)=r_{j}\left(y_{j}(t)\right)-r_{j}\left(\bar{y}_{j}(t)\right), \\
\tilde{f}_{l j}\left(v_{j}(t)\right)=f_{l j}\left(y_{j}(t)\right)-f_{l j}\left(\bar{y}_{j}(t)\right), \quad \tilde{g}_{l i}\left(u_{i}(t)\right)=g_{l i}\left(x_{i}(t)\right)-g_{l i}\left(\bar{x}_{i}(t)\right) .
\end{gathered}
$$

Theorem 3.1. If (A1)-(A6) hold, then the solutions of (2.1) are exponentially locally stable. That is the difference of any two solutions converges to zero exponentially provided that their initial data are close enough and we do not start from the equilibrium.

Proof. From (A1), (A4) and (A5), the upper right Dini derivatives $D^{+}\left|u_{i}(t)\right|$ and $D^{+}\left|v_{j}(t)\right|$ satisfy

$$
\begin{aligned}
D^{+}\left|u_{i}(t)\right| \leq & -\beta_{i}\left|u_{i}(t)\right|+\sum_{j \neq j_{0}} L_{1 j}\left|a_{j i}\right|\left|v_{j}(t)\right|+\sum_{j \neq j_{0}}\left|d_{j i}\right| L_{2 j} \int_{-\infty}^{t} k_{j i}(t-s)\left|v_{j}(s)\right| \mathrm{d} s \\
& +\left|a_{j_{0} i}\right|\left|v_{j_{0}}(t)\right| \tilde{f}_{1 j_{0}}\left(\left|v_{j_{0}}(t)\right|\right)+\int_{-\infty}^{t}\left|d_{j_{0} i}\right| k_{j_{0} i}(t-s)\left|v_{j_{0}}(s)\right| \tilde{f}_{2 j_{0}}\left(\left|v_{j_{0}}(s)\right|\right) \mathrm{d} s
\end{aligned}
$$

and

$$
\begin{aligned}
D^{+}\left|v_{j}(t)\right| \leq & -\bar{\beta}_{j}\left|v_{j}(t)\right|+\sum_{i \neq i_{0}} M_{1 i}\left|\bar{a}_{i j}\right|\left|u_{i}(t)\right|+\sum_{i \neq i_{0}}\left|\bar{d}_{i j}\right| M_{2 i} \int_{-\infty}^{t} h_{i j}(t-s)\left|u_{i}(s)\right| \mathrm{d} s \\
& +\left|\bar{a}_{i_{0} j}\right|\left|u_{i_{0}}(t)\right| \tilde{g}_{1 i_{0}}\left(\left|u_{i_{0}}(t)\right|\right)+\int_{-\infty}^{t}\left|\bar{d}_{i_{0} j}\right| h_{i_{0} j}(t-s)\left|u_{i_{0}}(s)\right| \tilde{g}_{2 i_{0}}\left(\left|u_{i_{0}}(s)\right|\right) \mathrm{d} s
\end{aligned}
$$

where the summations $\sum_{i=1, i \neq i_{0}}^{n}$ and $\sum_{j=1, j \neq j_{0}}^{m}$ are abbreviated by $\sum_{i \neq i_{0}}$ and $\sum_{j \neq j_{0}}$, resp. Then

$$
\begin{aligned}
D^{+} u(t) \leq & -\beta u(t)+\sum_{i=1}^{n} \sum_{j \neq j_{0}} L_{1 j}\left|a_{j i}\right|\left|v_{j}(t)\right|+\sum_{i=1}^{n} \sum_{j \neq j_{0}}\left|d_{j i}\right| L_{2 j} \int_{-\infty}^{t} k_{j i}(t-s)\left|v_{j}(s)\right| \mathrm{d} s \\
& +\sum_{i=1}^{n}\left|a_{j_{0} i}\right|\left|v_{j_{0}}(t)\right| \tilde{f}_{1 j_{0}}\left(\left|v_{j_{0}}(t)\right|\right)+\sum_{i=1}^{n}\left|d_{j_{0} i}\right| \int_{-\infty}^{t} k_{j_{0} i}(t-s)\left|v_{j_{0}}(s)\right| \tilde{f}_{2 j_{0}}\left(\left|v_{j_{0}}(s)\right|\right) \mathrm{d} s
\end{aligned}
$$


and

$$
\begin{aligned}
D^{+} v(t) \leq & -\bar{\beta} v(t)+\sum_{j=1}^{m} \sum_{i \neq i_{0}} M_{1 i}\left|\bar{a}_{i j}\right|\left|u_{i}(t)\right|+\sum_{j=1}^{m} \sum_{i \neq i_{0}}\left|\bar{d}_{i j}\right| M_{2 i} \int_{-\infty}^{t} h_{i j}(t-s)\left|u_{i}(s)\right| \mathrm{d} s \\
& +\sum_{j=1}^{m}\left|\bar{a}_{i_{0} j}\right|\left|u_{i_{0}}(t)\right| \tilde{g}_{1 i_{0}}\left(\left|u_{i_{0}}(t)\right|\right)+\sum_{j=1}^{m}\left|\bar{d}_{i_{0} j}\right| \int_{-\infty}^{t} h_{i_{0} j}(t-s)\left|u_{i_{0}}(s)\right| \tilde{g}_{2 i_{0}}\left(\left|u_{i_{0}}(s)\right|\right) \mathrm{d} s .
\end{aligned}
$$

Setting $P(t):=u(t)+v(t)$, the upper right Dini derivative of $P(t)$ fulfills

$$
\begin{aligned}
& D^{+} P(t) \leq-\left[\beta-\sum_{j=1}^{m} \sum_{i \neq i_{0}} M_{1 i}\left|\bar{a}_{i j}\right|\right] u(t)-\left[\bar{\beta}-\sum_{i=1}^{n} \sum_{j \neq j_{0}} L_{1 j}\left|a_{j i}\right|\right] v(t)+\sum_{i=1}^{n}\left|a_{j_{0} i}\right| v(t) \tilde{f}_{1 j_{0}}(v(t)) \\
& \quad+\sum_{j=1}^{m}\left|\bar{a}_{i_{0} j}\right| u(t) \tilde{g}_{1 i_{0}}(u(t))+\int_{-\infty}^{t} \sum_{i=1}^{n} \sum_{j=1}^{m}\left|d_{j i}\right| k_{j i}(t-s) v(s)\left[\max _{j \neq j_{0}} L_{2 j}+\tilde{f}_{2 j_{0}}(v(s))\right] \mathrm{d} s \\
& \quad+\int_{-\infty}^{t} \sum_{i=1}^{n} \sum_{j=1}^{m}\left|\bar{d}_{i j}\right| h_{i j}(t-s) u(s)\left[\max _{i \neq i_{0}} M_{2 i}+\tilde{g}_{2 i_{0}}(u(s))\right] \mathrm{d} s
\end{aligned}
$$

or

$$
\begin{aligned}
& D^{+} P(t) \leq-\left[\beta-\sum_{j=1}^{m} \sum_{i \neq i_{0}} M_{1 i}\left|\bar{a}_{i j}\right|\right] u(t)-\left[\bar{\beta}-\sum_{i=1}^{n} \sum_{j \neq j_{0}} L_{1 j}\left|a_{j i}\right|\right] v(t)+\sum_{i=1}^{n}\left|a_{j_{0} i}\right| P(t) \tilde{f}_{1 j_{0}}(P(t)) \\
& \quad+\sum_{j=1}^{m}\left|\bar{a}_{i_{0} j}\right| P(t) \tilde{g}_{1 i_{0}}(P(t))+\int_{-\infty}^{t} \sum_{i=1}^{n} \sum_{j=1}^{m}\left|d_{j i}\right| k_{j i}(t-s) \mid P(s)\left[\max _{j \neq j_{0}} L_{2 j}+\tilde{f}_{2 j_{0}}(P(s))\right] \mathrm{d} s \\
& \quad+\int_{-\infty}^{t} \sum_{i=1}^{n} \sum_{j=1}^{m}\left|\bar{d}_{i j}\right| h_{i j}(t-s) P(s)\left[\max _{i \neq i_{0}} M_{2 i}+\tilde{g}_{2 i_{0}}(P(s))\right] \mathrm{d} s .
\end{aligned}
$$

In a simpler form, we may write

$$
D^{+} P(t) \leq-A P(t)+F(P(t))+\int_{-\infty}^{t} H(t-s) G(P(s)) \mathrm{d} s
$$

where

$$
\begin{aligned}
A & =\min \left\{\beta-\sum_{j=1}^{m} \sum_{i \neq i_{0}} L_{1 j}\left|a_{j i}\right|, \bar{\beta}-\sum_{i=1}^{n} \sum_{j \neq j_{0}} M_{1 i}\left|\bar{a}_{i j}\right|\right\}, \\
F(P(t)) & =P(t) \tilde{F}(P(t)), \tilde{F}(P(t))=\max \left\{\sum_{i=1}^{n}\left|a_{j_{0} i}\right|, \sum_{j=1}^{m}\left|\bar{a}_{i_{0} j}\right|\right\}\left(\tilde{f}_{1 j_{0}}(P(t))+\tilde{g}_{1 i_{0}}(P(t))\right), \\
G(P(t)) & =P(t) \tilde{G}(P(t)), \tilde{G}(P(t))=\max _{j \neq j_{0}} L_{2 j}+\max _{i \neq i_{0}} M_{2 i}+\tilde{f}_{2 j_{0}}(P(t))+\tilde{g}_{2 i_{0}}(P(t)), \\
H_{i j}(t) & =\sup _{t \geq 0}\left\{\left|d_{j i}\right| k_{j i}(t),\left|\bar{d}_{i j}\right| h_{i j}(t)\right\}, H(t)=\sum_{i=1}^{n} \sum_{j=1}^{m} H_{i j}(t) .
\end{aligned}
$$

It is obvious that

$$
\tilde{G}(0)=\max _{j \neq j_{0}} L_{2 j}+\max _{i \neq i_{0}} M_{2 i}+\tilde{f}_{2 j_{0}}(0)+\tilde{g}_{2 i_{0}}(0) .
$$


By virtue of the assumption (A5), we find

$$
\tilde{G}(0)=\max _{j \neq j_{0}} L_{2 j}+\max _{i \neq i_{0}} M_{2 i}
$$

Next, from the assumption (A6), it holds that

$$
\tilde{G}(0) \int_{0}^{\infty} H(s) \mathrm{d} s<A .
$$

In view of the definition of $\tilde{F}$ and the assumption (A5), the inequality (3.6) yields

$$
\tilde{G}(0) \int_{0}^{\infty} H(s) \mathrm{d} s<A-\tilde{F}(0),
$$

with $\tilde{F}(0)=\max \left\{\sum_{i=1}^{n}\left|a_{j_{0} i}\right|, \sum_{j=1}^{m}\left|\bar{a}_{i_{0} j}\right|\right\}\left(\tilde{f}_{1 j_{0}}(0)+\tilde{g}_{1 i_{0}}(0)\right)$.

Thus, Lemma 2.6 may be applied: Therefore, there exist $\nu>0$ and $\alpha$ such that $0<\alpha<\alpha_{0}$ and

$$
P(t) \leq \sup _{-\infty<t \leq 0}\left(\sum_{i=1}^{n}\left|\phi_{i}(t)-\bar{\phi}_{i}(t)\right|+\sum_{j=1}^{m}\left|\varphi_{j}(t)-\bar{\varphi}_{j}(t)\right|\right) e^{-\alpha t}, t \geq 0,
$$

when $\sup _{-\infty<t \leq 0}\left(\sum_{i=1}^{n}\left|\phi_{i}(t)-\bar{\phi}_{i}(t)\right|+\sum_{j=1}^{m}\left|\varphi_{j}(t)-\bar{\varphi}_{j}(t)\right|\right) \leq \nu$.

This completes the proof.

For the second result, we need the assumption

(A7) We have

$$
\begin{aligned}
& \left(\sum_{i=1}^{n} \sum_{j \neq j_{0}} L_{1 j}\left|a_{j i}\right|+\sum_{j=1}^{m} \sum_{i \neq i_{0}} M_{1 i}\left|\bar{a}_{i j}\right|\right)+\sum_{i=1}^{n} \sum_{j \neq j_{0}} L_{2 j}\left|d_{j i}\right|\left(\int_{0}^{\infty} e^{\alpha_{0} s} k_{j i}(s) \mathrm{d} s\right) \\
& \quad+\sum_{j=1}^{m} \sum_{i \neq i_{0}} M_{2 i}\left|\bar{d}_{i j}\right|\left(\int_{0}^{\infty} e^{\alpha_{0} s} h_{i j}(s) \mathrm{d} s\right)<A .
\end{aligned}
$$

Theorem 3.2. The conclusion of Theorem 3.1 holds with the same hypotheses except (A6) replaced by (A 7).

Proof. According to estimations (3.4) and (3.5), one has

$$
\begin{aligned}
D^{+}\left|u_{i}(t)\right| \leq & -\beta_{i}\left|u_{i}(t)\right|+\sum_{j \neq j_{0}} L_{1 j}\left|a_{j i}\right|\left|v_{j}(t)\right|+\sum_{j \neq j_{0}}\left|d_{j i}\right| L_{2 j} \int_{-\infty}^{t} k_{j i}(t-s)\left|v_{j}(s)\right| \mathrm{d} s \\
& +\left|a_{j_{0} i}\right|\left|v_{j_{0}}(t)\right| \tilde{f}_{1 j_{0}}\left(\left|v_{j_{0}}(t)\right|\right)+\int_{-\infty}^{t}\left|d_{j_{0} i}\right| k_{j_{0} i}(t-s)\left|v_{j_{0}}(s)\right| \tilde{f}_{2 j_{0}}\left(\left|v_{j_{0}}(s)\right|\right) \mathrm{d} s
\end{aligned}
$$

and

$$
D^{+}\left|v_{j}(t)\right| \leq-\bar{\beta}_{j}\left|v_{j}(t)\right|+\sum_{i \neq i_{0}} M_{1 i}\left|\bar{a}_{i j}\right|\left|u_{i}(t)\right|+\sum_{i \neq i_{0}}\left|\bar{d}_{i j}\right| M_{2 i} \int_{-\infty}^{t} h_{i j}(t-s)\left|u_{i}(s)\right| \mathrm{d} s
$$




$$
+\left|\bar{a}_{i_{0} j}\right|\left|u_{i_{0}}(t)\right| \tilde{g}_{1 i_{0}}\left(\left|u_{i_{0}}(t)\right|\right)+\int_{-\infty}^{t}\left|\bar{d}_{i_{0} j}\right| h_{i_{0} j}(t-s)\left|u_{i_{0}}(s)\right| \tilde{g}_{2 i_{0}}\left(\left|u_{i_{0}}(s)\right|\right) \mathrm{d} s .
$$

We introduce the functionals, for $i=1,2, \ldots, n, j=1,2, \ldots, m$

$$
\begin{aligned}
& V_{1 i}(t)=e^{-\beta_{1} t} \int_{0}^{\infty} \int_{t-s}^{t} e^{\beta_{1}(s+\theta)}\left[\sum_{j \neq j_{0}}\left|d_{j i}\right| L_{2 j} k_{j i}(s)\left|v_{j}(\theta)\right|+\left|d_{j_{0} i}\right| k_{j_{0} i}(s)\left|v_{j_{0}}(\theta)\right| \tilde{f}_{2 j_{0}}\left(\left|v_{j_{0}}(\theta)\right|\right)\right] \mathrm{d} \theta \mathrm{d} s \\
& V_{2 j}(t)=e^{-\beta_{2} t} \int_{0}^{\infty} \int_{t-s}^{t} e^{\beta_{2}(s+\tau)}\left[\sum_{i \neq i_{0}}\left|\bar{d}_{i j}\right| M_{2 i} h_{i j}(s)\left|u_{i}(\tau)\right|+\left|\bar{d}_{i_{0} j}\right| h_{i_{0} j}(s)\left|u_{i_{0}}(\tau)\right| \tilde{g}_{2 i_{0}}\left(\left|u_{i_{0}}(\tau)\right|\right)\right] \mathrm{d} \tau \mathrm{d} s
\end{aligned}
$$

for some $0<\beta_{1}, \beta_{2}<\alpha_{0}$, and

$$
L(t):=u(t)+v(t)+V_{1}(t)+V_{2}(t), t \geq 0,
$$

where

$$
V_{1}(t)=\sum_{i=1}^{n} V_{1 i}(t), V_{2}(t)=\sum_{j=1}^{m} V_{2 j}(t)
$$

The differentiation of (3.10) and (3.11) for $t>0$ yields

$$
\begin{aligned}
V_{1 i}^{\prime}(t)= & -\beta_{1} V_{1 i}(t)+\sum_{j \neq j_{0}}\left|d_{j i}\right| L_{2 j}\left(\int_{0}^{\infty} e^{\beta_{1} s} k_{j i}(s) \mathrm{d} s\right)\left|v_{j}(t)\right| \\
& +\left|d_{j_{0} i}\right|\left(\int_{0}^{\infty} e^{\beta_{1} s} k_{j_{0} i}(s) \mid \mathrm{d} s\right)\left|v_{j_{0}}(t)\right| \tilde{f}_{2 j_{0}}\left(\left|v_{j_{0}}(t)\right|\right) \\
& -\int_{0}^{\infty}\left[\sum_{j \neq j_{0}}\left|d_{j i}\right| L_{2 j} k_{j i}(s)\left|v_{j}(t-s)\right|+\left|d_{j_{0} i}\right| k_{j_{0} i}(s)\left|v_{j_{0}}(t-s)\right| \tilde{f}_{2 j_{0}}\left(\left|v_{j_{0}}(t-s)\right|\right)\right] \mathrm{d} s
\end{aligned}
$$

and

$$
\begin{aligned}
V_{2 j}^{\prime}(t)= & -\beta_{2} V_{2 j}(t)+\sum_{i \neq i_{0}}\left|\bar{d}_{i j}\right| M_{2 i}\left(\int_{0}^{\infty} e^{\beta_{2} s} h_{i j}(s) \mid \mathrm{d} s\right)\left|u_{i}(t)\right| \\
& +\left|\bar{d}_{i_{0} j}\right|\left(\int_{0}^{\infty} e^{\beta_{2} s} h_{i_{0} j}(s) \mid \mathrm{d} s\right)\left|u_{i_{0}}(t)\right| \tilde{g}_{2 i_{0}}\left(\left|u_{i_{0}}(t)\right|\right) \\
& -\int_{0}^{\infty}\left[\sum_{i \neq i_{0}}\left|\bar{d}_{i j}\right| M_{2 i} h_{i j}(s)\left|u_{i}(t-s)\right|+\left|\bar{d}_{i_{0} j}\right| h_{i_{0} j}(s)\left|u_{i_{0}}(t-s)\right| \tilde{g}_{2 i_{0}}\left(\left|u_{i_{0}}(t-s)\right|\right)\right] \mathrm{d} s .
\end{aligned}
$$


Having in mind that, when the derivative exists, it is equal to all the Dini derivatives, from (3.8)-(3.14), the upper right Dini derivative of $L(t)$ satisfies

$$
\begin{aligned}
& D^{+} L(t) \leq-\beta u(t)-\beta_{1} V_{1}(t)-\beta_{2} V_{2}(t)-\bar{\beta} v(t)+\sum_{i=1}^{n} \sum_{j \neq j_{0}} L_{1 j}\left|a_{j i}\right|\left|v_{j}(t)\right|+\sum_{j=1}^{m} \sum_{i \neq i_{0}} M_{1 i}\left|\bar{a}_{i j}\right|\left|u_{i}(t)\right| \\
& \quad+\sum_{i=1}^{n} \sum_{j \neq j_{0}} L_{2 j}\left|d_{j i}\right| \int_{-\infty}^{t} k_{j i}(t-s)\left|v_{j}(s)\right| \mathrm{d} s+\sum_{i=1}^{n}\left|a_{j_{0} i}\right|\left|v_{j_{0}}(t)\right| \tilde{f}_{1 j_{0}}\left(\left|v_{j_{0}}(t)\right|\right) \\
& \quad+\sum_{i=1}^{n} \int_{-\infty}^{t}\left|d_{j_{0} i}\right| k_{j_{0} i}(t-s)\left|v_{j_{0}}(s)\right| \tilde{f}_{2 j_{0}}\left(\left|v_{j_{0}}(s)\right|\right) \mathrm{d} s+\sum_{j=1}^{m} \sum_{i \neq i_{0}} M_{2 i}\left|\bar{d}_{i j}\right| \int_{-\infty}^{t} h_{i j}(t-s)\left|u_{i}(s)\right| \mathrm{d} s \\
& \quad+\sum_{j=1}^{m}\left|\bar{a}_{i_{0} j}\right|\left|u_{i_{0}}(t)\right| \tilde{g}_{1 i_{0}}\left(\left|u_{i_{0}}(t)\right|\right)+\sum_{j=1}^{m} \int_{-\infty}^{t}\left|\bar{d}_{i_{0} j}\right| h_{i_{0} j}(t-s)\left|u_{i_{0}}(s)\right| \tilde{g}_{2 i_{0}}\left(\left|u_{i_{0}}(s)\right|\right) \mathrm{d} s \\
& \quad+\sum_{i=1}^{n} \sum_{j \neq j_{0}} L_{2 j}\left|d_{j i}\right|\left(\int_{0}^{\infty} e^{\beta_{1} s} k_{j i}(s) \mathrm{d} s\right)\left|v_{j}(t)\right|-\sum_{i=1}^{n} \sum_{j \neq j_{0}} \int_{0}^{\infty} L_{2 j}\left|d_{j i}\right| k_{j i}(s)\left|v_{j}(t-s)\right| \mathrm{d} s \\
& \quad+\sum_{i=1}^{n}\left|d_{j_{0} i}\right|\left(\int_{0}^{\infty} e^{\beta_{1} s} k_{j_{0} i}(s) \mathrm{d} s\right)\left|v_{j_{0}}(t)\right| \tilde{f}_{2 j_{0}}\left(\left|v_{j_{0}}(t)\right|\right)-\sum_{i=1}^{n}\left|d_{j_{0} i}\right| \int_{0}^{\infty} k_{j_{0} i}(s)\left|v_{j_{0}}(t-s)\right| \tilde{f}_{2 j_{0}}\left(\left|v_{j_{0}}(t-s)\right|\right) \mathrm{d} s \\
& \quad+\sum_{j=1}^{m} \sum_{i \neq i_{0}} M_{2 i}\left|\bar{d}_{i j}\right|\left(\int_{0}^{\infty} e^{\beta_{2} s} h_{i j}(s) \mathrm{d} s\right)\left|u_{i}(t)\right|+\sum_{j=1}^{m}\left|\bar{d}_{i_{0} j}\right|\left(\int_{0}^{\infty} e^{\beta_{2} s} h_{i_{0} j}(s) \mathrm{d} s\right)\left|u_{i_{0}}(t)\right| \tilde{g}_{2 i_{0}}\left(\left|u_{i_{0}}(t)\right|\right) \\
& \quad-\sum_{j=1}^{m} \sum_{i \neq i_{0}} \int_{0}^{\infty} M_{2 i}\left|\bar{d}_{i j}\right| h_{i j}(s)\left|u_{i}(t-s)\right| \mathrm{d} s-\sum_{j=1}^{m} \sum_{i \neq i_{0}} \int_{0}^{\infty}\left|\bar{d}_{i_{0} j}\right| h_{i_{0} j}(s)\left|u_{i_{0}}(t-s)\right| \tilde{g}_{2 i_{0}}\left(\left|u_{i_{0}}(t-s)\right|\right) \mathrm{d} s .
\end{aligned}
$$

This is simplified as

$$
\begin{aligned}
& D^{+} L(t) \leq-\beta u(t)-\beta_{1} V_{1}(t)-\bar{\beta} v(t)-\beta_{2} V_{2}(t)+\sum_{i=1}^{n} \sum_{j \neq j_{0}} L_{1 j}\left|a_{j i}\right|\left|v_{j}(t)\right| \\
& \quad+\sum_{i=1}^{n}\left|a_{j_{0}}\right|\left|v_{j_{0}}(t)\right| \tilde{f}_{1 j_{0}}\left(\left|v_{j_{0}}(t)\right|\right)+\sum_{j=1}^{m} \sum_{i \neq i_{0}} M_{1 i}\left|\bar{a}_{i j}\right|\left|u_{i}(t)\right|+\sum_{j=1}^{m}\left|\bar{a}_{i_{0} j}\right|\left|u_{i_{0}}(t)\right| \tilde{g}_{1 i_{0}}\left(\left|u_{i_{0}}(t)\right|\right) \\
& \quad+\sum_{i=1}^{n} \sum_{j \neq j_{0}} L_{2 j}\left|d_{j i}\right|\left(\int_{0}^{\infty} e^{\beta_{1} s} k_{j i}(s) \mathrm{d} s\right)\left|v_{j}(t)\right|+\sum_{i=1}^{n}\left|d_{j_{0} i}\right|\left(\int_{0}^{\infty} e^{\beta_{1} s} k_{j_{0} i}(s) \mathrm{d} s\right)\left|v_{j_{0}}(t)\right| \tilde{f}_{2 j_{0}}\left(\left|v_{j_{0}}(t)\right|\right) \\
& \quad+\sum_{j=1}^{m} \sum_{i \neq i_{0}} M_{2 i}\left|\bar{d}_{i j}\right|\left(\int_{0}^{\infty} e^{\beta_{2} s} h_{i j}(s) \mathrm{d} s\right)\left|u_{i}(t)\right|+\sum_{j=1}^{m}\left|\bar{d}_{i_{0} j}\right|\left(\int_{0}^{\infty} e^{\beta_{2} s} h_{i_{0} j}(s) \mathrm{d} s\right)\left|u_{i_{0}}(t)\right| \tilde{g}_{2 i_{0}}\left(\left|u_{i_{0}}(t)\right|\right),
\end{aligned}
$$

or

$$
\begin{aligned}
D^{+} L(t) \leq & \left.-\left[\beta-\left(\sum_{j=1}^{m} \sum_{i \neq i_{0}} M_{1 i}\left|\bar{a}_{i j}\right|\right)-\sum_{j=1}^{m} \sum_{i \neq i_{0}} M_{2 i}\left|\bar{d}_{i j}\right|\left(\int_{0}^{\infty} e^{\beta_{2} s} h_{i j}(s) \mathrm{d} s\right)\right)\right] u(t) \\
& -\left[\bar{\beta}-\left(\sum_{i=1}^{n} \sum_{j \neq j_{0}} L_{1 j}\left|a_{j i}\right|\right)-\sum_{i=1}^{n} \sum_{j \neq j_{0}} L_{2 j}\left|d_{j i}\right|\left(\int_{0}^{\infty} e^{\beta_{1} s} k_{j i}(s) \mathrm{d} s\right)\right] v(t) \\
& -\beta_{1} V_{1}(t)-\beta_{2} V_{2}(t)+\sum_{i=1}^{n}\left|a_{j_{0} i}\right|\left|v_{j_{0}}(t)\right| \tilde{f}_{1 j_{0}}\left(\left|v_{j_{0}}(t)\right|\right)+\sum_{j=1}^{m}\left|\bar{a}_{i_{0} j}\right|\left|u_{i_{0}}(t)\right| \tilde{g}_{1 i_{0}}\left(\left|u_{i_{0}}(t)\right|\right) \\
& +\sum_{i=1}^{n}\left|d_{j_{0} i}\right|\left(\int_{0}^{\infty} e^{\beta_{1} s} k_{j_{0} i}(s) \mathrm{d} s\right)\left|v_{j_{0}}(t)\right| \tilde{f}_{2 j_{0}}\left(\left|v_{j_{0}}(t)\right|\right) \\
& +\sum_{j=1}^{m}\left|\bar{d}_{i_{0} j}\right|\left(\int_{0}^{\infty} e^{\beta_{2} s} h_{i_{0} j}(s) \mathrm{d} s\right)\left|u_{i_{0}}(t)\right| \tilde{g}_{2 i_{0}}\left(\left|u_{i_{0}}(t)\right|\right) .
\end{aligned}
$$


We may further estimate $D^{+} L(t)$ as follows

$$
\begin{aligned}
D^{+} L(t) \leq & -\left[\beta-\left(\sum_{j=1}^{m} \sum_{i \neq i_{0}} M_{1 i}\left|\bar{a}_{i j}\right|\right)-\sum_{j=1}^{m} \sum_{i \neq i_{0}} M_{2 i}\left|\bar{d}_{i j}\right|\left(\int_{0}^{\infty} e^{\beta_{2} s} h_{i j}(s) \mathrm{d} s\right)\right] u(t) \\
& -\left[\bar{\beta}-\left(\sum_{i=1}^{n} \sum_{j \neq j_{0}} L_{1 j}\left|a_{j i}\right|\right)-\sum_{i=1}^{n} \sum_{j \neq j_{0}} L_{2 j}\left|d_{j i}\right|\left(\int_{0}^{\infty} e^{\beta_{1} s} k_{j i}(s) \mathrm{d} s\right)\right] v(t) \\
& -\beta_{1} V_{1}(t)-\beta_{2} V_{2}(t)+\left(\sum_{i=1}^{n}\left|a_{j_{0} i}\right|\right) L(t) \tilde{f}_{1 j_{0}}(L(t))+\left(\sum_{j=1}^{m}\left|\bar{a}_{i_{0} j}\right|\right) L(t) \tilde{g}_{1 i_{0}}(L(t)) \\
& +\tilde{f}_{2 j_{0}}(L(t))\left(\sum_{i=1}^{n}\left|d_{j_{0} i}\right|\left(\int_{0}^{\infty} e^{\beta_{1} s} k_{j_{0} i}(s) \mathrm{d} s\right)\right) L(t) \\
& +\tilde{g}_{2 i_{0}}(L(t))\left(\sum_{j=1}^{m}\left|\bar{d}_{i_{0} j}\right|\left(\int_{0}^{\infty} e^{\beta_{2} s} h_{i_{0} j}(s) \mathrm{d} s\right)\right) L(t),
\end{aligned}
$$

which we can write in short in the form

$$
D^{+} L(t) \leq-[A-F(L(t))] L(t)
$$

with

$$
\begin{aligned}
A= & \min \left\{\beta_{1}, \beta_{2}, \beta-\left(\sum_{j=1}^{m} \sum_{i \neq i_{0}} M_{1 i}\left|\bar{a}_{i j}\right|\right)-\sum_{j=1}^{m} \sum_{i \neq i_{0}} M_{2 i}\left|\bar{d}_{i j}\right|\left(\int_{0}^{\infty} e^{\beta_{2} s} h_{i j}(s) \mathrm{d} s\right),\right. \\
& \left.\bar{\beta}-\left(\sum_{i=1}^{n} \sum_{j \neq j_{0}} L_{1 j}\left|a_{j i}\right|\right)-\sum_{i=1}^{n} \sum_{j \neq j_{0}} L_{2 j}\left|d_{j i}\right|\left(\int_{0}^{\infty} e^{\beta_{1} s} k_{j i}(s) \mathrm{d} s\right)\right\}, \\
F(L(t))= & \left(\sum_{i=1}^{n}\left|a_{j_{0} i}\right|\right) \tilde{f}_{1 j_{0}}(L(t))+\tilde{f}_{2 j_{0}}(L(t))\left(\sum_{i=1}^{n}\left|d_{j_{0} i}\right|\left(\int_{0}^{\infty} e^{\beta_{1} s} k_{j_{0} i}(s) \mathrm{d} s\right)\right) \\
& +\left(\sum_{j=1}^{m}\left|\bar{a}_{i_{0} j}\right|\right) \tilde{g}_{1 i_{0}}(L(t))+\tilde{g}_{2 i_{0}}(L(t))\left(\sum_{j=1}^{m}\left|\bar{d}_{i_{0} j}\right|\left(\int_{0}^{\infty} e^{\beta_{2} s} h_{i_{0} j}(s) \mathrm{d} s\right)\right) .
\end{aligned}
$$

We shall compare solutions of (3.15) to solutions of the differential problem

$$
D^{+} z(t)=-[A-F(z(t))] z(t), \quad t>0,
$$

with

$$
\begin{aligned}
z(0)= & z_{0}:=L(0)=u(0)+v(0)+\sum_{i=1}^{n} \int_{0}^{\infty} \int_{-s}^{0} e^{\beta_{1}(s+\theta)}\left[\sum_{j \neq j_{0}}\left|d_{j i}\right| L_{2 j} k_{j i}(s)\left|\tilde{\varphi}_{j_{0}}(\theta)\right|\right. \\
& \left.+\left|d_{j_{0} i}\right| k_{j_{0} i}(s)\left|\tilde{\varphi}_{j_{0}}(\theta)\right| \tilde{f}_{2 j_{0}}\left(\left|\tilde{\varphi}_{j_{0}}(\theta)\right|\right)\right] \mathrm{d} \theta \mathrm{d} s+\sum_{j=1}^{m} \int_{0}^{\infty} \int_{-s}^{0} e^{\beta_{2}(s+\tau)}\left[\sum_{i \neq i_{0}}\left|\bar{d}_{i j}\right| M_{2 i} h_{i j}(s)\right. \\
& \left.+\left|\bar{d}_{i_{0} j} h_{i_{0} j}(s)\right| \tilde{\phi}_{i_{0}}(\tau) \mid \tilde{g}_{2 i_{0}}\left(\left|\tilde{\phi}_{i_{0}}(\tau)\right|\right)\right] \mathrm{d} \tau \mathrm{d} s
\end{aligned}
$$


Let

$$
p:=\sup \{\sigma \geq 0: F(\sigma)<A\}
$$

In light of our assumptions $\tilde{f}_{1 j_{0}}(0)=\tilde{f}_{2 j_{0}}(0)=\tilde{g}_{1 i_{0}}(0)=\tilde{g}_{2 i_{0}}(0)=0$, we have $p>0$. For $q, 0<q<p$, we define

$$
Q(\xi):=-A+\xi+F(q) .
$$

Due to the non-decreasingness of the function $F$ and $q<p$, then

$$
Q(0)=-A+F(q)<0
$$

Besides,

$$
\lim _{\xi \rightarrow \infty} Q(\xi)=\infty
$$

In view of (3.17) and (3.18), then for any positive real number $\lambda$ that satisfies $-\lambda>-A+F(q)$, one can find a $\xi_{\lambda}>0$ such that

$$
Q\left(\xi_{\lambda}\right):=-A+\xi_{\lambda}+F(q)=-\lambda<0 .
$$

We claim that, if $z_{0}<q$, then

$$
z(t) \leq q e^{-\xi_{\lambda} t}, t \geq 0
$$

We argue by contradiction. Assume that $t^{*}>0$ is the first time

$$
z(t)<q e^{-\xi_{\lambda} t}, 0 \leq t<t^{*}
$$

$z\left(t^{*}\right)=q e^{-\xi_{\lambda} t^{*}}$ and $\psi^{\prime}\left(t^{*}\right) \geq 0$, where $\psi(t):=z(t) e^{\xi_{\lambda} t}$. It is easy to see that

$$
\begin{aligned}
0 & \leq \psi^{\prime}\left(t^{*}\right)=\left.\left[z(t) e^{\xi_{\lambda} t}\right]^{\prime}\right|_{t=t^{*}}=z^{\prime}\left(t^{*}\right) e^{\xi_{\lambda} t^{*}}+\xi_{\lambda} z\left(t^{*}\right) e^{\xi_{\lambda} t^{*}} \\
& \leq e^{\xi_{\lambda} t^{*}}\left[-A z\left(t^{*}\right)+F\left(z\left(t^{*}\right)\right) z\left(t^{*}\right)\right]+\xi_{\lambda} z\left(t^{*}\right) e^{\xi_{\lambda} t^{*}} \\
& =\left(-A+\xi_{\lambda}+F\left(z\left(t^{*}\right)\right)\right) z\left(t^{*}\right) e^{\xi_{\lambda} t^{*}}=\left(-A+\xi_{\lambda}+F\left(z\left(t^{*}\right)\right)\right) \psi\left(t^{*}\right) \\
& \leq\left(-A+\xi_{\lambda}+F(q)\right) \psi\left(t^{*}\right)
\end{aligned}
$$

and therefore, from (3.16) and (3.19), we infer

$$
0 \leq \psi^{\prime}\left(t^{*}\right) \leq\left(-A+\xi_{\lambda}+F(q)\right) \psi\left(t^{*}\right)<0
$$

This contradiction confirms the relation (3.20). By comparison, we obtain

$$
L(t) \leq B e^{-\xi^{*} t}, \quad t \geq 0
$$


and thus

$$
u(t) \leq B e^{-\xi^{*} t}, \quad v(t) \leq B e^{-\xi^{*} t}, t \geq 0,
$$

for some positive constants $\xi^{*}$ and $B$. This completes the proof.

Remark 3.3. 1. We note that it is not necessary to verify the assumption

$$
\tilde{f}_{1 j_{0}}(0)=\tilde{f}_{2 j_{0}}(0)=\tilde{g}_{1 i_{0}}(0)=\tilde{g}_{2 i_{0}}(0)=0,
$$

we need in the nonlinear version of Halanay inequality (Lemma 2.6) the following estimation

$$
\tilde{G}(0) \int_{0}^{\infty} H(s) \mathrm{d} s<A-\tilde{F}(0),
$$

which implies

$$
\begin{aligned}
& {\left[\max _{j \neq j_{0}} L_{2 j}+\tilde{f}_{2 j_{0}}(0)\right] \sum_{i=1}^{n} \sum_{j=1}^{m}\left|d_{j i}\right| \int_{0}^{\infty} k_{j i}(s) \mathrm{d} s+\left[\max _{i \neq i_{0}} M_{2 i}+\tilde{g}_{2 i_{0}}(0)\right] \sum_{i=1}^{n} \sum_{j=1}^{m}\left|\bar{d}_{i j}\right| \int_{0}^{\infty} h_{i j}(s) \mathrm{d} s} \\
& \quad<\left[\beta-\sum_{j=1}^{m} \sum_{i \neq i_{0}} M_{1 i}\left|\bar{a}_{i j}\right|\right]+\left[\bar{\beta}-\sum_{i=1}^{n} \sum_{j \neq j_{0}} L_{1 j}\left|a_{j i}\right|\right]-\left(\sum_{i=1}^{n}\left|a_{j_{0} i}\right|\right) \tilde{f}_{1 j_{0}}(0)-\left(\sum_{j=1}^{m}\left|\bar{a}_{i_{0} j}\right|\right) \tilde{g}_{1 i_{0}}(0)
\end{aligned}
$$

for Theorem 3.1.

In the proof of Theorem 3.2, we need $F(0)<A$, which leads to

$$
\begin{aligned}
& \left(\sum_{i=1}^{n}\left|a_{j_{0} i}\right|\right) \tilde{f}_{1 j_{0}}(0)+\tilde{f}_{2 j_{0}}(0)\left(\sum_{i=1}^{n}\left|d_{j_{0} i}\right|\left(\int_{0}^{\infty} e^{\beta_{1} s} k_{j_{0} i}(s) \mathrm{d} s\right)\right) \\
& \quad+\left(\sum_{j=1}^{m}\left|\bar{a}_{i_{0} j}\right|\right) \tilde{g}_{1 i_{0}}(0)+\tilde{g}_{2 i_{0}}(0)\left(\sum_{j=1}^{m}\left|\bar{d}_{i_{0} j}\right|\left(\int_{0}^{\infty} e^{\beta_{2} s} h_{i_{0} j}(s) \mathrm{d} s\right)\right) \\
& \quad<\min \left\{\beta_{1}, \beta_{2}, \beta-\left(\sum_{j=1}^{m} \sum_{i \neq i_{0}} M_{1 i}\left|\bar{a}_{i j}\right|\right)-\sum_{j=1}^{m} \sum_{i \neq i_{0}} M_{2 i}\left|\bar{d}_{i j}\right|\left(\int_{0}^{\infty} e^{\beta_{2} s} h_{i j}(s) \mathrm{d} s\right),\right. \\
& \left.\quad \bar{\beta}-\left(\sum_{i=1}^{n} \sum_{j \neq j_{0}} L_{1 j}\left|a_{j i}\right|\right)-\sum_{i=1}^{n} \sum_{j \neq j_{0}} L_{2 j}\left|d_{j i}\right|\left(\int_{0}^{\infty} e^{\beta_{1} s} k_{j i}(s) \mathrm{d} s\right)\right\} .
\end{aligned}
$$

2. The obtained results improve the ones in [42, 43], where the conditions

$$
y f_{1 j_{0}}(y)>0, y f_{2 j_{0}}(y)>0, y g_{1 i_{0}}(y)>0, y g_{2 i_{0}}(y)>0, \text { for } y \neq 0
$$

and

$$
\sup _{y \neq 0} \frac{f_{1 j_{0}}(y)}{y}, \sup _{y \neq 0} \frac{f_{2 j_{0}}(y)}{y}, \sup _{y \neq 0} \frac{g_{1 i_{0}}(y)}{y}, \sup _{y \neq 0} \frac{g_{2 i_{0}}(y)}{y}
$$

corresponding to our functions $f_{1 j_{0}}(y), f_{2 j_{0}}(y), g_{1 i_{0}}(y), g_{2 i_{0}}(y)$ exist. 
3. The existence and uniqueness may be deduced from the following argument

$$
\begin{aligned}
\mid & \int_{-\infty}^{t_{1}} k_{j i}\left(t_{1}-s\right) f_{2 j}\left(y_{j}(s)\right) \mathrm{d} s-\int_{-\infty}^{t_{2}} k_{j i}\left(t_{2}-s\right) f_{2 j}\left(y_{j}(s)\right) \mathrm{d} s \mid \\
\leq & \left|\int_{-\infty}^{t_{1}} k_{j i}\left(t_{1}-s\right) f_{2 j}\left(y_{j}(s)\right) \mathrm{d} s-\int_{-\infty}^{t_{1}} k_{j i}\left(t_{2}-s\right) f_{2 j}\left(y_{j}(s)\right) \mathrm{d} s\right| \\
& +\left|\int_{-\infty}^{t_{1}} k_{j i}\left(t_{2}-s\right) f_{2 j}\left(y_{j}(s)\right) \mathrm{d} s-\int_{-\infty}^{t_{2}} k_{j i}\left(t_{2}-s\right) f_{2 j}\left(y_{j}(s)\right) \mathrm{d} s\right| \\
\leq & \int_{-\infty}^{t_{1}}\left|k_{j i}\left(t_{1}-s\right)-k_{j i}\left(t_{2}-s\right)\right|\left|f_{2 j}\left(y_{j}(s)\right)\right| \mathrm{d} s+\left|\int_{t_{2}}^{t_{1}} k_{j i}\left(t_{2}-s\right) f_{2 j}\left(y_{j}(s)\right) \mathrm{d} s\right|, \\
\mid & \int_{-\infty}^{t_{1}} h_{i j}\left(t_{1}-s\right) g_{2 i}\left(x_{i}(s)\right) \mathrm{d} s-\int_{-\infty}^{t_{2}} h_{i j}\left(t_{2}-s\right) g_{2 i}\left(x_{i}(s)\right) \mathrm{d} s \mid \\
\leq & \left|\int_{-\infty}^{t_{1}} h_{i j}\left(t_{1}-s\right) g_{2 i}\left(x_{i}(s)\right) \mathrm{d} s-\int_{-\infty}^{t_{1}} h_{i j}\left(t_{2}-s\right) g_{2 i}\left(x_{i}(s)\right) \mathrm{d} s\right| \\
& +\left|\int_{-\infty}^{t_{1}} h_{i j}\left(t_{2}-s\right) g_{2 i}\left(x_{i}(s)\right) \mathrm{d} s-\int_{-\infty}^{t_{2}} h_{i j}\left(t_{2}-s\right) g_{2 i}\left(x_{i}(s)\right) \mathrm{d} s\right| \\
\leq & \int_{-\infty}^{t_{1}}\left|h_{i j}\left(t_{1}-s\right)-h_{i j}\left(t_{2}-s\right)\right|\left|g_{2 i}\left(x_{i}(s)\right)\right| \mathrm{d} s+\left|\int_{t_{2}}^{t_{1}} h_{i j}\left(t_{2}-s\right) g_{2 i}\left(x_{i}(s)\right) \mathrm{d} s\right| .
\end{aligned}
$$

If $k_{j i}$ and $h_{i j}$ are Lipschitz and bounded, then

$$
\begin{aligned}
& \left|\int_{-\infty}^{t_{1}} k_{j i}\left(t_{1}-s\right) f_{2 j}\left(y_{j}(s)\right) \mathrm{d} s-\int_{-\infty}^{t_{2}} k_{j i}\left(t_{2}-s\right) f_{2 j}\left(y_{j}(s)\right) \mathrm{d} s\right| \\
& \leq K_{j i}\left|t_{1}-t_{2}\right| \int_{-\infty}^{t_{1}} f_{2 j}\left(y_{j}(s)\right) \mathrm{d} s+\Lambda\left|t_{1}-t_{2}\right|, \\
& \left|\int_{-\infty}^{t_{1}} h_{i j}\left(t_{1}-s\right) g_{2 i}\left(x_{i}(s)\right) \mathrm{d} s-\int_{-\infty}^{t_{2}} h_{i j}\left(t_{2}-s\right) g_{2 i}\left(x_{i}(s)\right) \mathrm{d} s\right| \\
& \leq H_{i j}\left|t_{1}-t_{2}\right| \int_{-\infty}^{t_{1}} g_{2 i}\left(x_{i}(s)\right) \mathrm{d} s+\bar{\Lambda}\left|t_{1}-t_{2}\right| .
\end{aligned}
$$

This, added to our a priori estimates, implies the Lipschitz continuity with respect to $t$ and Cid's Theorem 2.4 [4] implies the well-posedness.

4. The stability of the equilibrium needs additional conditions on the $i_{0}$ and $j_{0}$ components that ensure the uniqueness of the equilibrium (such as the Osgood condition). However, our argument will then depend on the existence of $\tilde{f}_{1 j_{0}}, \tilde{f}_{2 j_{0}}, \tilde{g}_{1 i_{0}}$ and $\tilde{g}_{2 i_{0}}$ (see (1.2)) fulfilling the conditions (see 2. in Rem. 3.3).

\section{NumERiCAL ILLUSTRATION}

In this section, we present a numerical example to validate the above results. Observe that, the Hölder continuous functions (having an exponent between 0 and 1) cannot be covered by our results. These were studied in [7, 27-33]. It is known that when the exponents exceed one, the functions are constant. Neither are the Log-Lipschitz continuous functions, for example, functions with $x|\ln (x)|$ as a continuity modulus (because $\ln (x)$ is unbounded near 0$)$. These functions do not satisfy the Lipschitz condition, whereas Osgood's condition 
is verified (which guarantees the uniqueness of the equilibrium). Nevertheless, these results can be applied to locally Lipschitz continuous functions.

Example 4.1. Consider the following BAM neural network system with distributed delays that contains four neurons

$$
\left\{\begin{array}{l}
x_{1}^{\prime}(t)=-c_{1}\left(x_{1}(t)\right)+\sum_{j=1}^{2} a_{j 1} f_{1 j}\left(y_{j}(t)\right)+\sum_{j=1}^{2} d_{j 1} \int_{0}^{t} k_{j 1}(s) f_{2 j}\left(y_{j}(t-s)\right) \mathrm{d} s+1.5, \quad t \in[0,5], \\
x_{2}^{\prime}(t)=-c_{2}\left(x_{2}(t)\right)+\sum_{j=1}^{2} a_{j 2} f_{1 j}\left(y_{j}(t)\right)+\sum_{j=1}^{2} d_{j 2} \int_{0}^{t} k_{j 2}(s) f_{2 j}\left(y_{j}(t-s)\right) \mathrm{d} s+1, \quad t \in[0,5] \\
y_{1}^{\prime}(t)=-r_{1}\left(y_{1}(t)\right)+\sum_{i=1}^{2} \bar{a}_{i 1} g_{1 i}\left(x_{i}(t)\right)+\sum_{i=1}^{2} \bar{d}_{i 1} \int_{0}^{t} h_{i 1}(s) g_{2 i}\left(x_{i}(t-s)\right) \mathrm{d} s+2, \quad t \in[0,5] \\
y_{2}^{\prime}(t)=-r_{2}\left(y_{2}(t)\right)+\sum_{i=1}^{2} \bar{a}_{i 2} g_{1 i}\left(x_{i}(t)\right)+\sum_{i=1}^{2} \bar{d}_{i 2} \int_{0}^{t} h_{i 2}(s) g_{2 i}\left(x_{i}(t-s)\right) \mathrm{d} s+0.5, \quad t \in[0,5]
\end{array}\right.
$$

where $a_{11}=0.12, a_{12}=0.23, a_{21}=0.5, a_{22}=1, d_{11}=0.5, d_{12}=0.25, d_{21}=0.5, d_{22}=2.5, \bar{a}_{11}=$ $0.25, \bar{a}_{12}=0.2, \bar{a}_{21}=0.75, \bar{a}_{22}=1.5, \bar{d}_{11}=\frac{1}{3}, \bar{d}_{12}=1, \bar{d}_{21}=\frac{1}{3}, \bar{d}_{22}=2, c_{1}(x)=4 x, c_{2}(x)=7 x, r_{1}(x)=$ $5 x, r_{2}(x)=8 x, f_{11}(x)=f_{21}(x)=\frac{1}{2}(|x+1|-|x-1|), g_{11}(x)=g_{21}(x)=\tanh (x), f_{12}(x)=x^{2}, f_{22}(x)=$ $x^{3}, g_{12}(x)=x^{2}+1, g_{22}(x)=x^{3}+2, k_{j i}(t)=e^{-5 t}, h_{i j}(t)=e^{-6 t}, x_{1}(t)=1, x_{2}(t)=1.5, y_{1}(t)=2, y_{2}(t)=$ $3, t \in[-5,0]$.

Taking $\alpha_{0}=4, \beta_{1}=\beta_{2}=3, i_{0}=j_{0}=2$. Through simple calculations, one can obtain $\beta=4, \bar{\beta}=5, L_{1 j}=$ $L_{2 j}=M_{1 i}=M_{2 i}=1, \int_{0}^{\infty} e^{\alpha_{0} t} k_{j i}(t) \mathrm{d} t=1, \int_{0}^{\infty} e^{\alpha_{0} t} h_{i j}(t) \mathrm{d} t=0.5$.

Besides, assumptions (A6) and (A7) are satisfied

$$
\begin{gathered}
\left(\max _{i \neq i_{0}} M_{2 i}+\max _{j \neq j_{0}} L_{2 j}\right) \int_{0}^{\infty} H(t) \mathrm{d} t=1.64 \\
<\min \left\{\beta-\sum_{i=1}^{2} \sum_{j \neq j_{0}} L_{1 j}\left|a_{j i}\right|, \bar{\beta}-\sum_{j=1}^{2} \sum_{i \neq i_{0}} M_{1 i}\left|\bar{a}_{i j}\right|\right\}=3.65
\end{gathered}
$$

and

$$
\begin{gathered}
\left(\sum_{i=1}^{2} \sum_{j \neq j_{0}} L_{1 j}\left|a_{j i}\right|+\sum_{j=1}^{2} \sum_{i \neq i_{0}} M_{1 i}\left|\bar{a}_{i j}\right|\right)+\sum_{i=1}^{2} \sum_{j \neq j_{0}} L_{2 j}\left|d_{j i}\right|\left(\int_{0}^{\infty} e^{\alpha_{0} s} k_{j i}(s) \mathrm{d} s\right) \\
+\sum_{j=1}^{2} \sum_{i \neq i_{0}} M_{2 i}\left|\bar{d}_{i j}\right|\left(\int_{0}^{\infty} e^{\alpha_{0} s} h_{i j}(s) \mathrm{d} s\right)=2.21<A=3 .
\end{gathered}
$$

The difference between solutions $(x(t), y(t))$ and $(\bar{x}(t), \bar{y}(t))$ of system (4.1) is given by

$$
u_{i}(t)=x_{i}(t)-\bar{x}_{i}(t), i=1,2, \quad v_{j}(t)=y_{j}(t)-\bar{y}_{j}(t), j=1,2
$$

and satisfies 


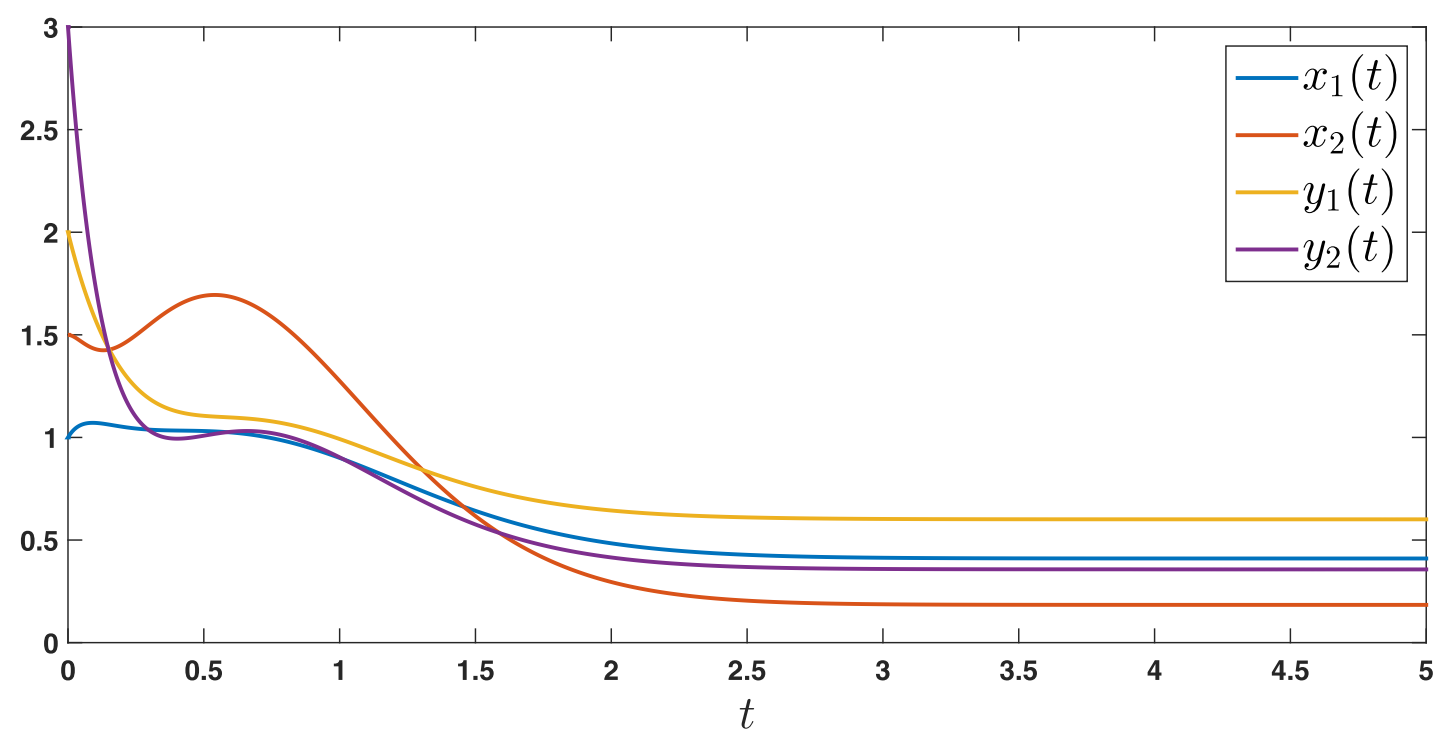

Figure 1. Trajectories of the states $x_{1}(t), x_{2}(t), y_{1}(t)$ and $y_{2}(t)$.

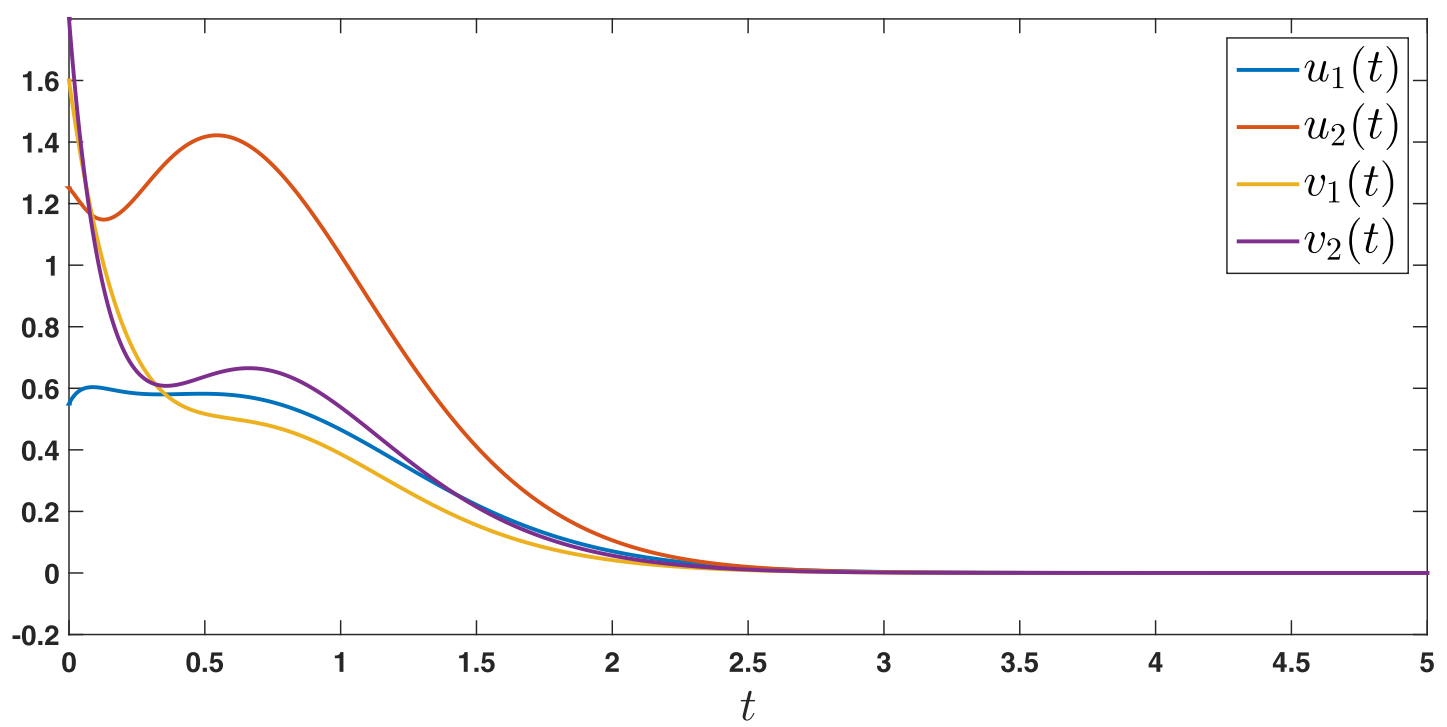

Figure 2. Trajectories of the states $u_{1}(t), u_{2}(t), v_{1}(t)$ and $v_{2}(t)$.

\footnotetext{
$D^{+} u_{1}(t) \leq-\left(c_{1}\left(x_{1}(t)\right)-c_{1}\left(\bar{x}_{1}(t)\right)\right)+\left|a_{11}\right|\left|v_{1}(t)\right|+\left|a_{21}\right|\left|v_{2}(t)\right|\left|\tilde{f}_{12}\left(y_{2}(t), \bar{y}_{2}(t)\right)\right|$

$+\left|d_{11}\right| \int_{-\infty}^{t} k_{11}(t-s)\left|v_{1}(s)\right| \mathrm{d} s+\left|d_{21}\right| \int_{-\infty}^{t} k_{21}(t-s)\left|v_{2}(s)\right|\left|\tilde{f}_{22}\left(y_{2}(s), \bar{y}_{2}(s)\right)\right| \mathrm{d} s, t \in[0,5]$,

$D^{+} u_{2}(t) \leq-\left(c_{2}\left(x_{2}(t)\right)-c_{2}\left(\bar{x}_{2}(t)\right)\right)+\left|a_{12}\right|\left|v_{1}(t)\right|+\left|a_{22}\right|\left|v_{2}(t)\right|\left|\tilde{f}_{12}\left(y_{2}(t), \bar{y}_{2}(t)\right)\right|$

$+\left|d_{12}\right| \int_{-\infty}^{t} k_{12}(t-s)\left|v_{1}(s)\right| \mathrm{d} s+\left|d_{22}\right| \int_{-\infty}^{t} k_{22}(t-s)\left|v_{2}(s)\right|\left|\tilde{f}_{22}\left(y_{2}(s), \bar{y}_{2}(s)\right)\right| \mathrm{d} s, t \in[0,5]$,

$D^{+} v_{1}(t) \leq-\left(r_{1}\left(y_{1}(t)\right)-r_{1}\left(\bar{y}_{1}(t)\right)\right)+\left|\bar{a}_{11}\right|\left|u_{1}(t)\right|+\left|\bar{a}_{21}\right|\left|u_{2}(t)\right|\left|\tilde{g}_{12}\left(x_{2}(t), \bar{x}_{2}(t)\right)\right|$

$+\left|\bar{d}_{11}\right| \int_{-\infty}^{t} h_{11}(t-s)\left|u_{1}(s)\right| \mathrm{d} s+\left|\bar{d}_{21}\right| \int_{-\infty}^{t} h_{21}(t-s)\left|u_{2}(s)\right|\left|\tilde{g}_{22}\left(x_{2}(s), \bar{x}_{2}(s)\right)\right| \mathrm{d} s, t \in[0,5]$,

$D^{+} v_{1}(t) \leq-\left(r_{2}\left(y_{2}(t)\right)-r_{2}\left(\bar{y}_{2}(t)\right)\right)+\left|\bar{a}_{12}\right|\left|u_{1}(t)\right|+\left|\bar{a}_{22}\right|\left|u_{2}(t)\right|\left|\tilde{g}_{12}\left(x_{2}(t), \bar{x}_{2}(t)\right)\right|$

$+\left|\bar{d}_{12}\right| \int_{-\infty}^{t} h_{12}(t-s)\left|u_{1}(s)\right| \mathrm{d} s+\left|\bar{d}_{22}\right| \int_{-\infty}^{t} h_{22}(t-s)\left|u_{2}(s)\right|\left|\tilde{g}_{22}\left(x_{2}(s), \bar{x}_{2}(s)\right)\right| \mathrm{d} s, t \in[0,5]$,

$u_{1}(t)=0.5, \quad u_{2}(t)=1.25, t \in[-5,0]$,

$v_{1}(t)=1.6, \quad v_{2}(t)=1.8, t \in[-5,0]$,
} 


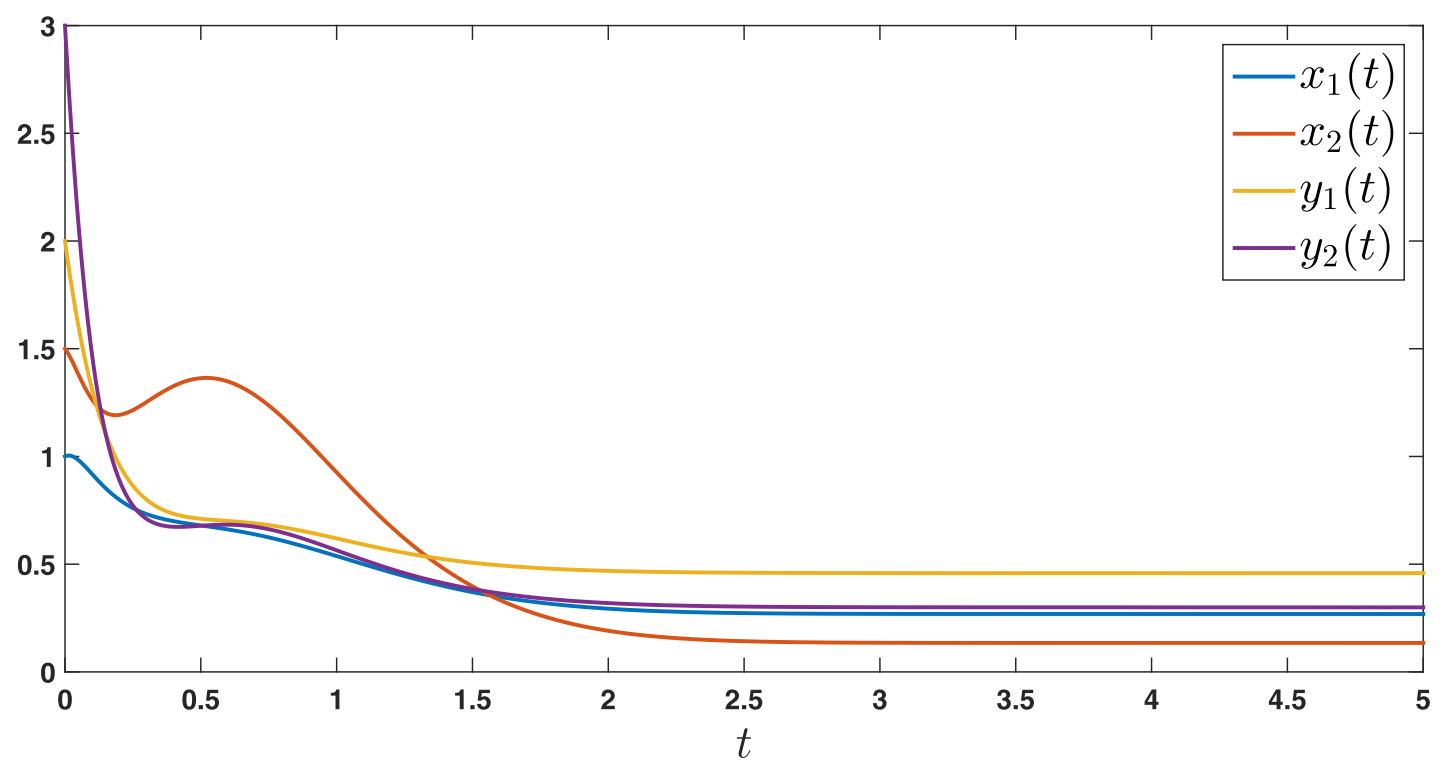

FIgURE 3 . Trajectories of the states $x_{1}(t), x_{2}(t), y_{1}(t)$ and $y_{2}(t)$.

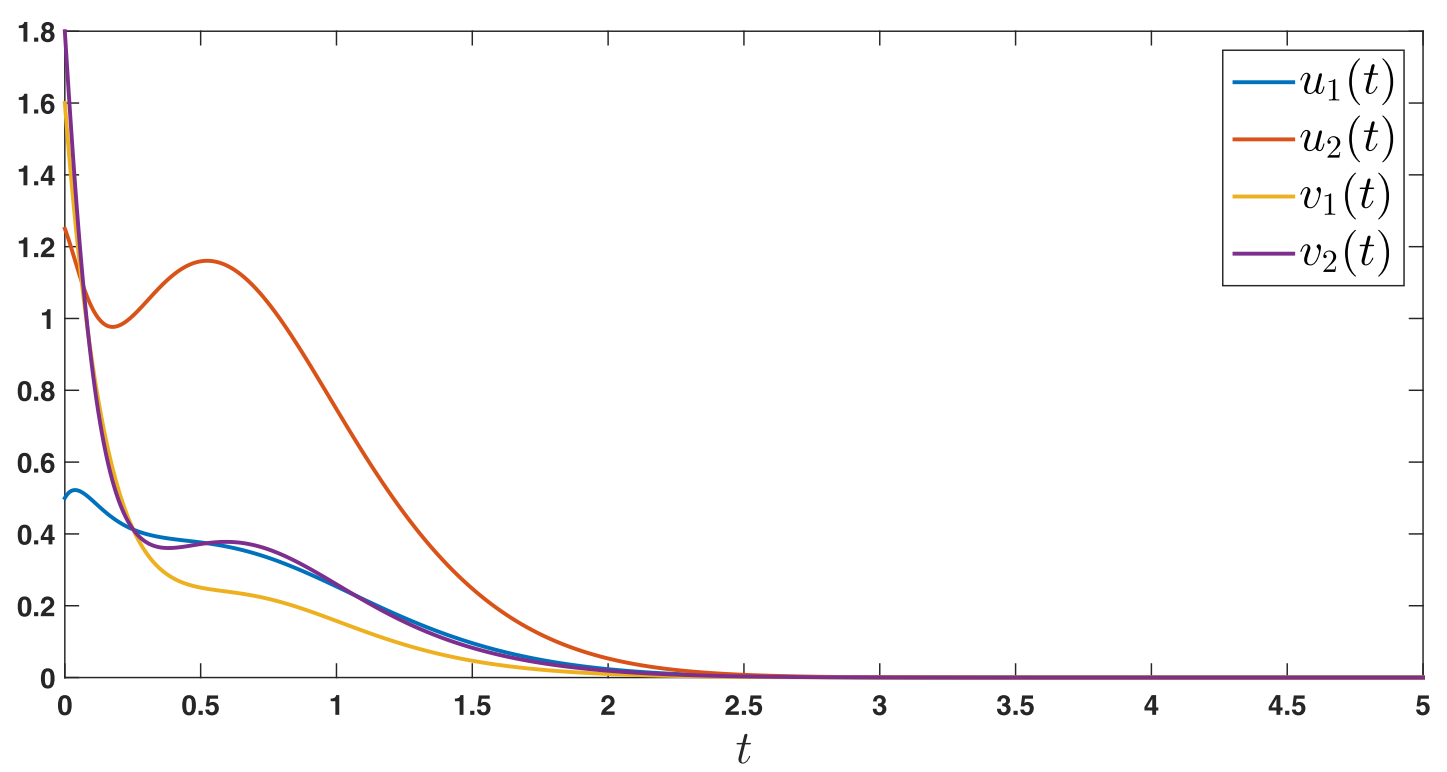

Figure 4 . Trajectories of the states $u_{1}(t), u_{2}(t), v_{1}(t)$ and $v_{2}(t)$.

where

$$
\begin{array}{ll}
\tilde{f}_{12}\left(y_{2}(t), \bar{y}_{2}(t)\right)=y_{2}(t)+\bar{y}_{2}(t), & \tilde{f}_{22}\left(y_{2}(t), \bar{y}_{2}(t)\right)=y_{1}^{2}(t)+y_{2}(t) \bar{y}_{2}(t)+\bar{y}_{2}^{2}(t), \\
\tilde{g}_{12}\left(x_{2}(t), \bar{x}_{2}(t)\right)=x_{2}(t)+\bar{x}_{2}(t), & \tilde{g}_{22}\left(x_{2}(t), \bar{x}_{2}(t)\right)=\bar{x}^{2}(t)+x_{2}(t) \bar{x}_{2}(t) .
\end{array}
$$

As, assumptions (A1) $-(\mathbf{A 7})$ are met, Theorems 3.1 and 3.2 imply that solutions of system (4.1) are exponentially stable. This is depicted by Figures 1 and 2 . 
Example 4.2. Consider system (4.1) of Example 4.1 with the same parameters and functions. The functions $c_{1}(x), c_{2}(t), r_{1}(x)$ and $r_{2}(x)$ are selected as $c_{1}(x)=5 x+x \cos (x), c_{2}(x)=8 x+x \cos (x), r_{1}(x)=6 x+$ $x \sin (x), r_{2}(x)=9 x+x \sin (x)$. By choosing the same parameters as in Example 4.1, the assumptions (A1)(A7) are satisfied, and via Theorems 3.1 and 3.2, solutions of system (4.1) are exponentially stable. This is shown in Figures 3 and 4.

Acknowledgements. The second author would like to thank King Fahd University of Petroleum and Minerals for its continuous support through project IN 171006 (Interdisciplinary Research Center for Intelligent Manufacturing \& Robotics). We would like to express our appreciation to the referees for their helpful comments and suggestions.

\section{REFERENCES}

[1] P. Arena, A. Basile, M. Bucolo and L. Fortuna, Image processing for medical diagnosis using CNN. Nucl. Instruments Methods Phys. Res. Sect. A 497 (2003) 174-178.

[2] G. Bao and Z. Zeng, Analysis and design of associative memories based on recurrent neural network with discontinuous activation functions. Neurocomputing $\mathbf{7 7}$ (2012) 101-107.

[3] Z. Cai and L. Huang, Existence and global asymptotic stability of periodic solution for discrete and distributed time-varying delayed neural networks with discontinuous activations. Neurocomputing $\mathbf{7 4}$ (2011) 3170-3179.

[4] J.A. Cid, On uniqueness criteria for systems of ordinary differential equations. J. Math. Anal. Appl. 281 (2003) $264-275$.

[5] E.Y. Cong, X. Han and X. Zhang, Global exponential stability analysis of discrete-time BAM neural networks with delays: A mathematical induction approach. Neurocomputing 379 (2020) 227-235.

[6] B.T. Cui and X.Y. Lou, Global asymptotic stability of BAM neural networks with distributed delays and reaction-diffusion terms. Chaos Solitons Fractals 27 (2006) 1347-1354.

[7] M. Forti, M. Grazzini, P. Nistri and L. Pancioni, Generalized Lyapunov approach for convergence of neural networks with discontinuous or non-Lipschitz activations. Phys. D Nonlinear Phenom. 214 (2006) 88-99.

[8] D. Gao and J. Li, Global asymptotic stability of periodic solutions for neutral-type BAM neural networks with delays. Neural Process. Lett. 51 (2020) 367-382.

[9] P. Hartman, Ordinary Differential Equations. Wiley, New York (1964).

[10] L. Van Hien and L.D. Hai-An, Exponential stability of positive neural networks in bidirectional associative memory model with delays. Math. Methods Appl. Sci. 42 (2019) 6339-6357.

[11] Y. Huang, H. Zhang and Z. Wang, Dynamical stability analysis of multiple equilibrium points in time-varying delayed recurrent neural networks with discontinuous activation functions. Neurocomputing 91 (2012) 21-28.

[12] M. Iswarya, R. Raja, G. Rajchakit, J. Cao, J. Alzabut and C. Huang, Existence, uniqueness and exponential stability of periodic solution for discrete-time delayed bam neural networks based on coincidence degree theory and graph theoretic method. Mathematics 7 (2019) 1-18.

[13] B. Kosko, Adaptive bi-directional associative memories. Appl. Optim. 26 (1987) 4947-4960.

[14] B. Kosko, Bi-directional associative memories. IEEE Trans. Syst. Man Cybern. 18 (1988) 49-60.

[15] B. Kosko, Neural networks and Fuzzy systems - a dynamical system approach machine intelligence. Englewood Cliffs, PrenticeHall (1992) 38-108.

[16] X. Li and J. Jia, Global robust stability analysis for BAM neural networks with time-varying delays. Neurocomputing 120 (2013) 499-503.

[17] Y. Li, L. Yang and W. Wu, Anti-periodic solution for impulsive BAM neural networks with time-varying leakage delays on time scales. Neurocomputing 149 (2015) 536-545.

[18] B. Liu, Global exponential stability for BAM neural networks with time-varying delays in the leakage terms. Nonlinear Anal. Real World Appl. 14 (2013) 559-566.

[19] Y. Liu, Z. Wang and X. Liu, Global asymptotic stability of generalized bi-directional associative memory networks with discrete and distributed delays. Chaos Solitons Fractals 28 (2006) 793-803.

[20] C. Maharajan, R. Raja, J. Cao, G. Rajchakit, Z. Tu and A. Alsaedi, LMI-based results on exponential stability of BAM-type neural networks with leakage and both time-varying delays: A non-fragile state estimation approach. Appl. Math. Comput. 326 (2018) 33-55.

[21] G. Mathai and B.R. Upadhyaya, Performance analysis and application of the bidirectional associative memory to industrial spectral signatures. Proc. IJCNN. 89 (1989) 33-37.

[22] S. Mohamad, K. Gopalsamy and H. Akça, Exponential stability of artificial neural networks with distributed delays and large impulses. Nonlinear Anal. Real World Appl. 9 (2008) 872-888.

[23] W. Peng, Q. Wu and Z. Zhang, LMI-based global exponential stability of equilibrium point for neutral delayed BAM neural networks with delays in leakage terms via new inequality technique. Neurocomputing 199 (2016) 103-113.

[24] Z. Quan, L. Huang, S. Yu and Z. Zhang, Novel LMI-based condition on global asymptotic stability for BAM neural networks with reaction-diffusion terms and distributed delays. Neurocomputing 136 (2014) 213-223.

[25] V. Sharma, R. Jha and R. Naresh, Optimal multi-reservoir network control by augmented Lagrange programming neural network. Appl. Soft Comput. J. 7 (2007) 783-790. 
[26] Q. Song, Novel criteria for global exponential periodicity and stability of recurrent neural networks with time-varying delays. Chaos Solitons Fractals 36 (2008) 720-728.

[27] N.-e. Tatar, Control of systems with Hölder continuous functions in the distributed delays. Carpathian J. Math. 30 (2014) $123-128$.

[28] N.-e. Tatar, Long time behavior for a system of differential equations with non-Lipschitzian nonlinearities. Adv. Artificial Neural Network. Syst. 2014 (2014) Article ID 252674.

[29] N.-e. Tatar, Haraux type activation functions in neural network theory. Br. J. Math. Math. Computer Sci. 4 (2014) 3163-3170.

[30] N.-e. Tatar, Neural networks with delayed Hölder continuous activation functions. Int. J. Artificial Intelligence Mech. 2 (2014) $156-160$.

[31] N.-e. Tatar, Hölder continuous activation functions. Adv. Diff. Eqs. Control Processes Neural Netw. 15 (2015) $93-106$.

[32] N.-e. Tatar, Exponential decay for a system of equations with distributed delays. J. Appl. Math. 2015 (2015) Article ID 981383.

[33] N.-e. Tatar, On a general nonlinear problem with distributed delays. J. Contemporary Math. Anal. 52 (2017) $184-190$.

[34] N.-e. Tatar, A nonlinear version of the distributed Halanay inequality and application. Submitted.

[35] H. Wu and C. Shan, Stability analysis for periodic solution of BAM neural networks with discontinuous neuron activations and impulses. Appl. Math. Model. 33 (2009) 2564-2574.

[36] H. Wu, F. Tao, L. Qin, R. Shi and L. He, Robust exponential stability for interval neural networks with delays and non-Lipschitz activation functions. Nonlinear Dyn. 66 (2011) 479-487.

[37] $\mathrm{H}$. Wu and X. Xue, Stability analysis for neural networks with inverse Lipschitzian neuron activations and impulses. Appl. Math. Model. 32 (2008) 2347-2359.

[38] C. Xu and P. Li, Existence and exponentially stability of anti-periodic solutions for neutral BAM neural networks with time-varying delays in the leakage terms. J. Nonlinear Sci. Appl. 9 (2016) 1285-1305.

[39] C. Xu, P. Li and Y. Pang, Existence and exponential stability of almost periodic solutions for neutral-type BAM neural networks with distributed leakage delays. Math. Methods Appl. Sci. 40 (2017) 2177-2196.

[40] Y. Yao, W.J. Freeman, B. Burke and Q. Yang, Pattern recognition by a distributed neural network: an industrial application. Neural Netw. 4 (1991) 103-121.

[41] H. Zhao, Global stability of bidirectional associative memory neural networks with distributed delays. Phys. Lett. A 297 (2002) $182-190$.

[42] H. Zhao, Global asymptotic stability of Hopfield neural networks involving distributed delays. Neural Networks $\mathbf{1 7}$ (2004) $47-53$.

[43] J. Zhou, S. Li and Z. Yang, Global exponential stability of Hopfield neural networks with distributed delays. Appl. Math. Model. 33 (2009) 1513-1520. 